\title{
On the stress recovery behaviour of Ecoflex silicone rubbers
}

\author{
Zisheng Liao ${ }^{\mathrm{a}, \mathrm{b}, \mathrm{c}}$, Jie Yang ${ }^{\mathrm{c}}$, Mokarram Hossain $^{\mathrm{b}, *}$, Gregory Chagnon $^{\mathrm{d}}$, Lin Jing $^{\mathrm{a}}$, Xiaohu Yao $^{\mathrm{a}, \mathrm{c}, *}$ \\ ${ }^{a}$ State Key Laboratory of Traction Power, Southwest Jiaotong University, Chengdu 610031, China \\ ${ }^{b}$ Zienkiewicz Centre for Computational Engineering, College of Engineering, Swansea University, SA1 8EN, United Kingdom \\ ${ }^{c}$ State Key Laboratory of Subtropical Building Science, South China University of Technology, Guangzhou 510640, China \\ ${ }^{d}$ University of Grenoble Alpes, CNRS, Grenoble INP, TIMC, F-38000 Grenoble, France
}

\begin{abstract}
Silicone rubbers are promising materials that have extensively been used in many areas, including wearable electronic devices, actuator materials in soft robotics, and energy harvesters. Ecoflex, a commercially available polymer, is a group of silicone rubbers appearing with various Shore hardnesses that has become popular in recent years. While silicone rubbers in their real applications are subjected to repeated loadingunloading conditions, their mechanical behaviour under cyclic loading in different deformation modes and the corresponding stress recovery behaviour are yet to be well investigated. In this contribution, we conduct loading-unloading cyclic experiments in different time gaps between the first cycle and the second cycle to demonstrate the stress recovery behaviour after stress softening happens during the first cycle. For that, three different modes of deformations, i.e., uniaxial tension, planar tension, and equibiaxial tension tests are selected. Besides, Shore hardness dependence with different strain levels is also taken into consideration. The results show that the stress softening could recover significantly with time. These findings will help to understand the mechanical behaviour of Ecoflex silicone rubbers before selecting them in practical applications that are exposed to repeated loading-unloading cycles.
\end{abstract}

Keywords: Ecoflex silicone rubber, Stress recovery, Shore hardness, Deformation mode

\section{Introduction}

Silicone rubbers attract considerable attention in recent years thanks to their wide range of promising and unique properties. For example, non-toxicity, non-reactiveness, and biocompatibility of silicone rubbers avoid rejection and inflammation when implanted in human tissues, and therefore facilitate the unrivalled applications in bio-medical accessories and devices [1, 2, 3]. Moreover, the matchable stiffnesses of silicones with biological tissues place them as ideal candidates for the use as substrates in mechanobiology, see $[4,5]$. Silicone sealants are also massively used in the industry thanks to their good thermal stability over a wide range of temperature, UV resistance, antioxidative properties, and high hydrophobicity [6, 7]. The electrical insulation also brings about the usage of silicone rubbers in electronic and electrical devices and apparatus [8]. More recently, silicone composites are increasingly utilised in several emerging sectors such as soft robotics $[9,10,11,12,13]$, flexible electronics [14, 15], and energy harvesters $[16,17,18]$ due to their large stretchability, reduced dissipative behaviour and other thermal, physical, mechanical, and

\footnotetext{
${ }^{*}$ Corresponding authors

Email addresses: 1.zisheng@mail.scut.edu.cn (Zisheng Liao), ct jyang@mail.scut.edu.cn (Jie Yang), mokarram. hossain@swansea.ac.uk (Mokarram Hossain), gregory . chagnon@univ-grenoble-alpes.fr (Gregory Chagnon), jinglineswjtu.edu.cn (Lin Jing), yaoxh@scut.edu.cn (Xiaohu Yao)
} 
biological behaviour.

Ecoflex ${ }^{\mathrm{TM}}$ polymers (Smooth-On, USA), a group of commercially available silicone rubbers with several Shore hardnesses, e.g., 00-50, 00-30, 00-20, 00-10, and so on, are typical elastomers for a variety of practical applications in recent years. They are two-component platinum-catalysed silicones cured at room temperature with negligible shrinkage and a relatively short curing time ranging from few minutes to several hours. Thanks to the high stretchability and durability, Ecoflex rubbers have widely been used in many fields such as cushioning, prosthetic appliance, wearable strain sensors for epidermal electronic systems with the mechanical compliance close to human skin. For example, several researchers designed and manufactured single-wall or multi-wall carbon nanotube (CNT)-Ecoflex nanocomposite thin films as soft strain sensors [15, 19, 20, 21], dielectric elastomer actuators [22], and smart keypad [23] with good sensitivity and flexibility. Other types of composites were demonstrated such as graphene foam/Ecoflex [26], and starch/Ecoflex/biomass waste composite green materials [27]. Ecoflex had also been applied to BaTiO3/Ecoflex-based piezoelectric nanogenerators for self-powered human motion monitoring [28] and multifunctional flexible piezo/triboelectric hybrid water energy harvesters based on biocompatible AlN and soft parylene-PDMS-Ecoflex [18].

In applications such as wearable sensors and energy harvest devices made of silicone elastomers, most components are subjected to multiple cyclic loads that considerably influence the mechanical behaviour, giving rise to the so-called Mullins effect or the stress softening. In the case of filled rubbers, Mullins [29] found that the stress of a material during reloading decreases significantly compared to the stress of the first loading at the same strain level. It is noteworthy that the stress softening is not permanent. The stress of the pre-stretched material can recover to the initial level after keeping it with stress-free state at room temperature or annealing it at an elevated temperature for a certain time, as Mullins [29] discussed. The stress softening and recovery show that the mechanical behaviour of silicone components keeps changing during the application and therefore influences the performance of the appliance to a certain extent. To this end, comprehensive investigation of the mechanical behaviour with respect to the stress softening and stress recovery is necessary. After Mullins' work in 1948 [29], the effect was researched extensively via both experimental studies [30, 31, 32] and mathematical modelling procedures [33, 34, 35, 36, 37, 38]. Some works also considered about softening behaviour of different deformation modes and the softening induced anisotropy, see for example [35, 39].

Since many studies dealing with experiments and constitutive modelling assume that the Mullins effect is a type of irreversible damage, the investigation of the stress recovery from the stress softening is relatively rare. After Mullins' findings [29], Rigbi [40] reported that at room temperature, carbon-black-filled rubbers could recover partially four weeks after the first cycle. Hanson et al. [30] reported a similar finding for silica-filled polydimethylsiloxane (PDMS) after a rest period of up to half a year. Yan et al. [41] also reported a complete recovery of the Mullins effect of the nanoparticle-filled PDMS polymer under a simple shear loading condition. In addition, the recovery of the Mullins effect has also been observed by Zheng et al. [42] in the tough gels with a faster speed than filled rubbers.

One of the ways to accelerate the stress recovery of polymeric materials is to anneal them at elevated temperatures. Increasing the annealing temperature plays a similar role as increasing the recovery time. Laraba-Abbes et al. [43] demonstrated that carbon-black filled rubbers recover completely after 48-hour 
annealing at $95^{\circ} \mathrm{C}$. Similarly, Diani et al. [36] observed a complete recovery of a carbon-black filled styrene-butadiene-rubber (SBR) after annealing it for 17 hours at $80^{\circ} \mathrm{C}$. Amin et al. [44] also found the stress recovery in the blend of natural rubber (NR) and polybutadiene rubber. Plagge and Klüppel [45] reported the temperature-induced recovery on non-crystallising rubbers including Ethylene-propylene-diene (EPDM), SBR, and hydrated-nitrile-butadiene rubber (HNBR) and well as strain-crystallising rubbers such as NR filled with different amounts of carbon black and silica. In addition to the recovery by annealing, NR can also recover from the Mullins effect by swelling in solvents, as investigated by Harwood and Payne [31].

The physical interpretations of the Mullins effect and its recovery are also extensively investigated in the literature. Diani et al. [36] gave an overview of physical interpretations and constitutive models of the Mullins effect. Diaz et al. [46] investigated the physical change in the microstructure of a carbon-black filled SBR and claimed that the stress softening is caused mainly by the desorption of chains at the filler an elevated temperature. Li et al. [47] analysed the energy dissipation and its recovery of the carbon-black filled nitrile butadiene rubber (NBR) nanocomposites and also found that the softening and the slow recovery process is due to the retardation of the rubber chain related to the filler. Later on, Li et al. [48] also found that the softening and its recovery are also dependent on filler types, temperature and the crosslinking agents. Several constitutive models for capturing the stress recovery from the Mullins effect are proposed in the literature. For instance, Drozdov and Dorfmann [49] developed a framework combining permanent and temporary networks to capture the Mullins effect as well as its recovery caused by thermal annealing and solvent exposure. Wang and Chester [34] developed a phenomenologically-motivated thermo-mechanically coupled constitutive model that captures the thermal recovery of the Mullins effect of three NR vulcanizates obtained from Harwood and Payne [31]. Very recently, Chu et al. [50] incorporated the micromechanical concepts of the dynamic scission and recovery of polymer chains to model the Mullins effect and its annealing recovery in the case of filled rubbers such as EPDM and silicones.

To the best of the authors' knowledge, the investigations of the mechanical behaviour of Ecoflex are limited

in the literature. Sparks et al. [51] conducted unconfined compression experiments on Ecoflex 00-10 and 00-30 as well as indentation tests on Ecoflex 00-30 to mimic the stress and strain distribution in deep muscle tissues and shed light on how deep tissue injury disrupts blood flow and metabolic clearance. Park et al. [52] compared several silicone elastomers, including Ecoflex, Dragon Skin, and Sylgard etc., and their blended compositions for the applications of highly stretchable and deformable microfluidic devices synthesizd using a soft lithography technique. Vaicekauskaite et al. [53] also investigated the mechanical and electrical properties of the blends of commercial silicone elastomers, including Ecoflex 00-50, 00-30, 00-10 and Sylgard 184, 186, for the fabrication of specific transducers. Very recently, Liao et al. [32] conducted systematic experimentation on Ecoflex 00-30 to characterise the thermo-mechanical behaviour including cyclic loading-unloading behaviour at various strain rates and temperature and relaxation tests at different strain levels with different pre-treatment. It is found that this material not only shows significant stress softening behaviour in subsequent cycles, but also reveals stress recovery behaviour at different waiting time after the pre-treatment. Liao et al. [38] extended the research to other Shore hardnesses including 00-50, 00-35, 00-30, 00-20, 00-10, and discovered that all these materials share similar mechanical behaviour including the stress softening and the stress recovery behaviour.

As mentioned above, cyclic loads in complex deformation modes are often applied to the silicone components resulting in stress softening. The stress recovery of the softening often involves a long time span that 
could significantly influence the subsequent mechanical behaviour of the silicone-based components. Therefore, sufficient research on the stress recovery behaviour of Ecoflex rubbers is required to understand how this behaviour is influenced by different factors. However, very few investigations into the stress recovery behaviour of the Ecoflex are carried out in the literature concerning the different recovery time, deformation modes, strain levels, Shore hardness. To make up for the absence of these researches, in this manuscript, we carry out a comprehensive investigation on the stress recovery behaviour of Ecoflex series silicone rubber, considering the factors mentioned above. The details of the experimental schedule will be discussed in Section 2.3. This manuscript is organised into five sections. Apart from the current section, the Section 2 elaborates the preparation work including the material and the specimen preparation, the experimental set-up and the schedule for experiments that are carried out. The following Section 3 illustrated our comprehensive experimentation in detail with discussion and analysis in consideration of factors including strain levels in Section 3.2, Section 3.3, Section 3.4, deformation modes in Section 3.5, and Shore hardness in Section 3.6. Finally, the conclusions of our major contributions in this study are drawn in the final Section 4. Some supplementary information is put in Appendix A.

\section{Experimental preliminaries}

\subsection{Material and specimen preparations}

In this contribution, four different Shore hardnesses, i.e., 00-50, 00-30, 00-20, 00-10, of Ecoflex are adopted for the experimental investigation. Ecoflex appears in two-component forms in which a platinum catalyst is added to cure the polymer. Specimens are prepared following the procedures as detailed in our previous works $[32,38]$. The two liquid components with a 1:1 volumetric ratio are mixed and stirred to make them uniform. The mixed liquids are injected into specimen moulds, where the weights of the specimens are Although the curing times for four types of Shore hardness are three to four hours as per the supplier guidelines, all specimens are demoulded smoothly after 24 hours for sufficient curing. To minimise the slippage, aluminium sheets are bonded to the grip sections of all specimens. When the specimens are put to rest after unloading for the recovery, they are put in ziplock bags storing in the lab so that the temperature can be controlled by the air conditioner at $22^{\circ} \mathrm{C}$

In this manuscript, the materials are tested under three different in-plane modes of deformations, i. e., uniaxial tension (UT), planar tension (PT), and equibiaxial tension (ET), which represent the simple tension, pure shear, and simple compression state of the materials, respectively [54, 55, 56, 57, 58]. As silicone rubbers are considered as incompressible materials, the product of three principle stretches is equal to identity, i.e., $\lambda_{1} \lambda_{2} \lambda_{3}=1$. Considering in-plane deformations, we can take $\lambda_{1}=\lambda$ as the axial direction, $\lambda_{2}$ as the orthogonal in-plane direction, and $\lambda_{3}$ as the direction perpendicular to the plane. For uniaxial tension, both transverse directions contracted freely and equally, i.e., $\lambda_{2}=\lambda_{3}=\lambda^{-1 / 2}$. For planar tension, the in-plane transverse deformation is restricted, i.e., $\lambda_{2}=1$, and the remaining principal stretch reads $\lambda_{3}=\lambda^{-1}$. For equibiaxial tension, both principal in-plane stretch are the same, i.e., $\lambda_{1}=\lambda_{2}=\lambda$, and therefore, $\lambda_{3}=\lambda^{-2}$. These three deformation modes can be represented by a biaxiality ratio $\mu$ [39], defined as

$$
\mu=\frac{\ln \left(\lambda_{\min }\right)}{\ln \left(\lambda_{\max }\right)},
$$

where, $\lambda_{\min }$ and $\lambda_{\max }$ are the maximum principal in-pane stretch and the minimum principal in-pane stretch, 


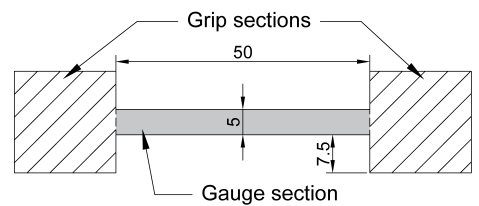

(a)

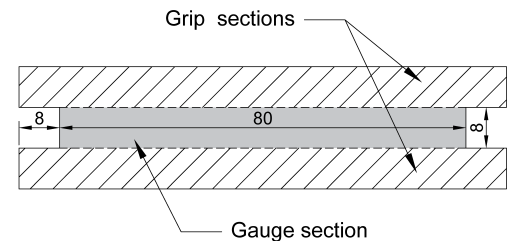

(b)

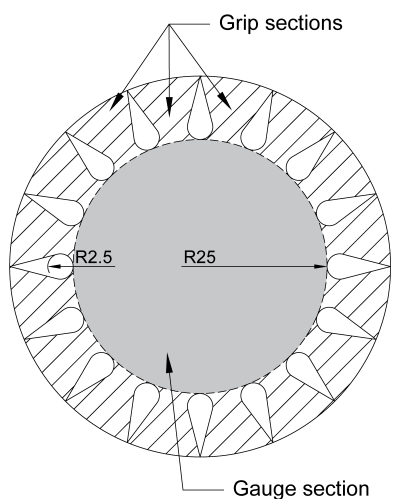

(c)

Figure 1. The shapes and dimensions of three types of specimens utilised in this manuscript (in mm). (a) Uniaxial tension, (b) planar tension, (c) equibiaxial tension.

respectively. Here, $\lambda_{\max }=\lambda_{1}$ and $\lambda_{\min }=\lambda_{2}$. We can easily achieve that the biaxiality ratio of uniaxial, planar and equibiaxial tension modes are $-1 / 2,0$, and 1 , respectively.

The shapes and sizes of specimens for the three different cases are depicted in Figure 1. All specimens adopt a $1 \mathrm{~mm}$ thickness that is small enough to exclude the influence of thickness on the mechanical responses. Specimens for the uniaxial tension tests adopt a 10:1 (length to width) rectangular shape of the gauge section as Figure 1(a) shows to ensure that no transverse constraint takes place in the main part of the specimens (c.f. [35, 59]). In contrast, the specimens for the planar tension tests adopt a 1:10 (length to width) rectangular shape as is shown in Figure 1(b) to ensure that no transverse contraction happens in the width direction (c.f. [35, 60]). In the case of equibiaxial tension tests, according to [61], three types of experiments are often used, including loading a cross-shape or a rectangular specimen in both the perpendicular axes (e.g. $[62,63])$, inflating a circular plane membrane into a "bubble" shape by air (e.g., [35]), and loading an axisymmetric specimen in radial directions (e.g., [56]). Here, we adopt the last technique. The specimens utilise a disk shape with sixteen grip sections surrounding the circumference, as shown in Figure 1(c). Such an axisymmetric geometry enables an approximately homogeneous radial deformation state in the main part of the specimen. The length or diameter of the gauge section is used for the calculation of stress and strain. The detailed calculation of the stress for a disk shape specimen can be found in [56]. The information of the deformation homogeneity can be found in Appendix A.

\subsection{Experimental set-up}

The comprehensive experimental study on the stress recovery behaviour of Ecoflex rubber is conducted by a testing system called Instron 5567 universal test machine. The machine frame can move up to $800 \mathrm{~mm}$ with a maximum crosshead speed of $8 \mathrm{~mm} / \mathrm{s}$. The loading conditions are controlled by the Instron built-in software, Bluehill ${ }^{\circledR}$. The testing machine equips with two force sensors with the capacity of $\pm 500 \mathrm{~N}$ and $\pm 50 \mathrm{~N}$, respectively, which are used for this study. In addition, specific grippers for different deformation modes are equipped. Note that the Digital Image Correlation (DIC) technique is adopted to capture the deformation of specimens more accurately, see Appendix A. 


\subsection{Experimental schedule}

The stress recovery behaviour of Ecoflex can be investigated by the two-cycle loading-unloading tests, see Figure 2 in Section 3.1. In our previous studies [32, 38], we observe that the first loading-unloading cycle applied on a virgin Ecoflex specimen triggers the stress softening (Mullins effect), where the stress level during the subsequent reloading cycle decreases significantly. However, the stress softening is not permanent. An increment of the stress, i.e. the stress recovery, is observed when the specimen is stored at the stress-free state for a certain amount of time between the two cycles. For a further investigation of the stress recovery, the two-cycle loading-unloading tests are carried out with regard to factors that can influence the recovery behaviour. The factors considered here are the dependence of recovery time varying from 0 second, 1 hour, 1 day (24 hours), 1 week (168 hours), 1 month ( 720 hours), and up to 1 year ( 8760 hours); the dependence of the strain level from $160 \%$ to $400 \%$ for different deformation modes (see Section 3.2, Section 3.3 Section 3.4); the dependence of the deformation modes including uniaxial tension, planar tension, and equibiaxial tension (see Section 3.5); the dependence of the Shore hardness including 00-10, 00-20, 00-30 and 00-50 (see Section 3.6). In consideration of these factors, the experiments at ambient temperature are planned as in Table 1. Note that all tests are carried out under the same strain rate of $0.1 / \mathrm{s}$, as we found the influence of the strain rate variation is relatively small for Ecoflex, see Liao et al. [32].

Table 1. The schedule of the recovery experiments at room temperature. $\bullet$ Uniaxial tension, $\bullet$ Planar tension, -Equibiaxial tension.

\begin{tabular}{|c|c|c|c|c|c|c|c|}
\hline \multirow{2}{*}{$\begin{array}{c}\text { Shore } \\
\text { hardness }\end{array}$} & \multirow{2}{*}{$\begin{array}{l}\text { Strain } \\
\text { level }\end{array}$} & \multicolumn{6}{|c|}{ Recovery time } \\
\hline & & $0 \mathrm{sec}$ & $1 \mathrm{hr}$ & 1 day & $1 \mathrm{wk}$ & $1 \mathrm{mon}$ & $3 \mathrm{mon} / 1 \mathrm{yr}$ \\
\hline \multirow{4}{*}{$00-50$} & $400 \%$ & - & • & • & & • & \\
\hline & $300 \%$ & $\bullet$ & $\bullet$ & $\bullet$ & & $\bullet$ & \\
\hline & $200 \%$ & - & $\bullet$ & $\bullet$ & & - & \\
\hline & $240 \%$ & $\bullet$ & & $\bullet$ & & $\bullet$ & \\
\hline \multirow{6}{*}{$00-30$} & $400 \%$ & $\bullet$ & $\bullet$ & $\bullet$ & $\bullet$ & $\bullet$ & $\bullet$ \\
\hline & $320 \%$ & $\bullet$ & $\bullet$ & $\bullet$ & & $\bullet$ & \\
\hline & $300 \%$ & • & • & $\bullet$ & • & $\bullet$ & - \\
\hline & $240 \%$ & $\bullet$ & $\bullet$ & $\bullet$ & & $\bullet$ & \\
\hline & $200 \%$ & $\bullet \bullet$ & $\bullet \bullet \bullet$ & $\bullet \bullet$ & $\bullet$ & $\bullet \bullet$ & - \\
\hline & $160 \%$ & $\bullet$ & $\bullet$ & $\bullet$ & & $\bullet$ & \\
\hline \multirow{4}{*}{$00-20$} & $400 \%$ & $\bullet$ & $\bullet$ & $\bullet$ & & $\bullet$ & \\
\hline & $300 \%$ & - & $\bullet$ & $\bullet$ & & $\bullet$ & \\
\hline & $200 \%$ & $\bullet$ & • & - & & $\bullet$ & \\
\hline & $240 \%$ & $\bullet$ & & $\bullet$ & & $\bullet$ & \\
\hline \multirow{4}{*}{$00-10$} & $400 \%$ & $\bullet$ & $\bullet$ & $\bullet$ & & $\bullet$ & \\
\hline & $300 \%$ & $\bullet$ & $\bullet$ & $\bullet$ & & $\bullet$ & \\
\hline & $200 \%$ & - & • & • & & $\bullet$ & \\
\hline & $240 \%$ & - & & - & & - & \\
\hline
\end{tabular}

All experimental results will be presented using nominal stresses (also known as the first Piola-Kirchhoff stresses) and nominal strains (also known as the engineering strains). In addition, the time derivatives of the nominal strains are termed strain rates. Good reproducibility of experimental results is of great importance. 
Hence, we repeat each condition of the experiments at least five times. The mean value, as well as the standard deviations, are calculated.

\section{Results and discussion}

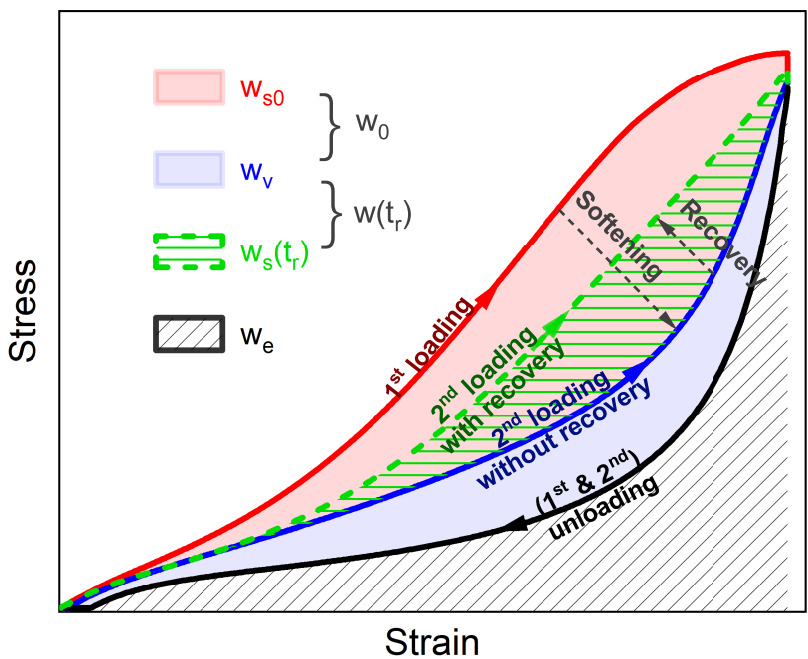

Figure 2. Schematic of the stress-strain response of two-cycle loading-unloading tests with and without recovery in between. The initial softening dissipation $\left(w_{s 0}\right)$, the subsequent softening dissipation $\left(w_{s}\right)$, the viscoelastic dissipation $\left(w_{v}\right)$, and the elastic energy $\left(w_{e}\right)$ are illustrated by various coloured areas.

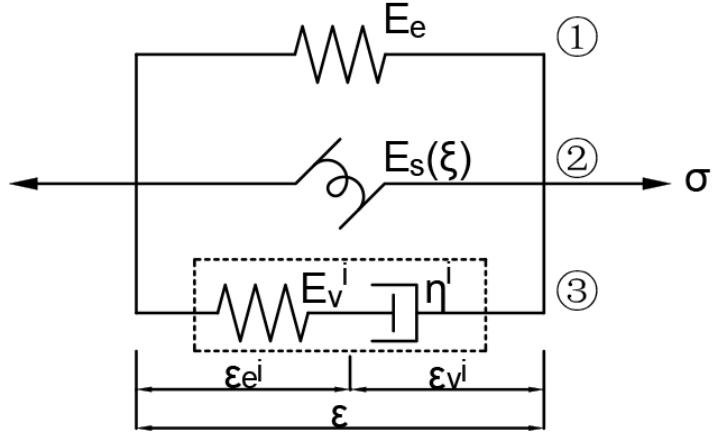

Figure 3. Schematic of a rheological model illustrating the macro-mechanical behaviour of the stress softening and recovery of Eooflex.

As is mentioned above, $\mathrm{Li}$ et al. [47] found that the total energy dissipation could be decomposed into a so-called recovery hysteresis part that related to viscoelasticity (termed as the viscoelastic dissipation in this manuscript) and a softening part related to the stress softening and stress recovery (termed as the softening dissipation). To decouple the viscoelasticity and stress softening and characterise the stress recovery behaviour of Ecoflex, the two-cycle loading-unloading tests are carried out. As the schematic Figure 2 shows, each complete test consists of two cycles of loading-unloading under the same experimental condition, i.e., the same deformation modes, strain rates, strain levels, etc. In-between the two cycles, the specimen is stored at a stress-free state for a certain duration (termed as the recovery time, $t_{r}$ ). Here we define the enclosed areas of the loop of a cycle, which represents the energy loss, as the dissipation energy density $w$, i.e.,

$$
w=\int_{0}^{t} \mathbf{P}: \dot{\mathbf{F}} \mathrm{d} t
$$

where, $\mathbf{P}$ is the nominal stress, and $\mathbf{F}$ is the deformation gradient. If we only consider the work done by the axial force, the dissipation yields

$$
w=\int_{l} \sigma \mathrm{d} \varepsilon
$$

where, $l$ is the loading-unloading path of a cycle, and $\sigma$ and $\varepsilon$ are the nominal stress and nominal strain, respectively. The first cycle is applied to a virgin specimen. We denote the total dissipation energy density 
enclosed by the first loading (the red curve in Figure 2) and the unloading (the black curve) as $w_{0}$ (the sum of the red and the blue regions in Figure 2). The first cycle triggers the stress softening and therefore any subsequent loading will demonstrate an obvious stress decrease compared with the initial value. When the subsequent cycle is applied immediately after the first cycle, i.e., $t_{r}=0$, the stress softening is just evacuated and the recovery time is minimised. Therefore, it can be roughly assumed that no stress recovery occurs on this loading path (the blue curve), and the dissipation is mainly contributed by the viscoelastic dissipation energy density $w_{v}=w\left(t_{r}=0\right)$, as the blue region illustrated in Figure 2. With different recovery time $t_{r}>0$ such as 1 hour, 1 day, 1 month, etc., the subsequent cycle (the second cycle) is then performed. An increase of stress in the second loading path with stress recovery (the green curve) and an increase of dissipation energy density $w\left(t_{r}\right)$ (enclosed by the green curve and the black curve) can be seen with the increase of recovery time. As the total dissipation is combined by the softening and viscoelastic dissipation, we can decouple initial softening dissipation energy density $w_{s 0}$, the softening part of dissipation in the first cycle, by $w_{s 0}=w_{0}-w_{v}$, as the red region showed in Figure 2. Similarly, the subsequent softening dissipation energy density, the softening part recovery during $t_{r}$, can also be decoupled by $w_{s}\left(t_{r}\right)=w\left(t_{r}\right)-w_{v}$, as the green (shaded) region shows. Note that our previous studies $[32,38]$ demonstrated that the unloading path of the first cycle and the second cycle (regardless of the recovery time) are superposed since the stress softening has taken place and mainly the elastic response remains, as the black curve shows.

These mechanical properties can be interpreted using a rheological model as illustrated in Figure 3 with three different elements arranged in parallel. Each element stands for a different physical nature. The first elements $E_{e}$ is a hyperelastic spring contributed by the stretchability of chains. It represents the entropic change of the rubber matrix (the polymer backbone) during deformation, where the energy potential corresponds to $w_{e}$ (black region in Figure 2). The second element $E_{s}$ is a damageable spring, standing for the stress softening and the stress recovery behaviour. It corresponds to the red region $w_{s 0}$ and the green region $w_{s}\left(t_{r}\right)$ in Figure 2. According to the literature [36], apart from crystallising natural polymers, stress softening takes place mainly in filled elastomers. Note that Krpovic et al. confirmed that Ecoflex silicone rubbers are filled polymers [64]. It is proven that the interaction of fillers and the rubber phase is the main source of the stress softening $[46,47]$. The fillers in the matrix create obstacles to the materials flow and therefore provide reinforcement [65]. During deformation, the desorption of polymer chains in the fillers surface happens gradually, leading to the degradation of fillers-rubber surface and energy dissipation. As a result, the reinforcement is disconnected, and on the macroscopic level, stress softening takes place. However, the rubber matrix can be absorbed again to the fillers' surfaces with time after unloading, as the network is moving towards the equilibrium conformation. It means that the stress softening is reversible. The third element is a general Maxwell element that consists of springs $E_{v}^{i}$ and dashpots $\eta^{i}$. This element represents the viscoelasticity corresponding to the blue region $w_{v}$ in Figure 2. It is known that the viscoelasticity is originated from the rubber phase [47, 66]. Considering an imperfect network of the matrix due to offstoichiometry reaction during processing, dangling chains and a sol fraction are formed with more freedom than the crosslinked chain network [66]. With a large molecular weight, entanglements tend to be formed in the matrix, behaving as 'crosslinks' that can slide [47, 66, 67]. These structures cause internal friction and energy dissipation during deformation. The internal friction leads to the time lag between deformation and stress response, and therefore the macroscopic time-dependent behaviours such as rate dependence, relaxation, creeping and strain recovery of the residual strain. Different from the softening part that can be removed, the viscoelasticity part still exists after cycles but can be removed after swelling to untrapped the entanglements as solute the sol fraction[47]. The combination of these three sections yields the complete stress response and the total energy accumulation of a filled polymeric material. 


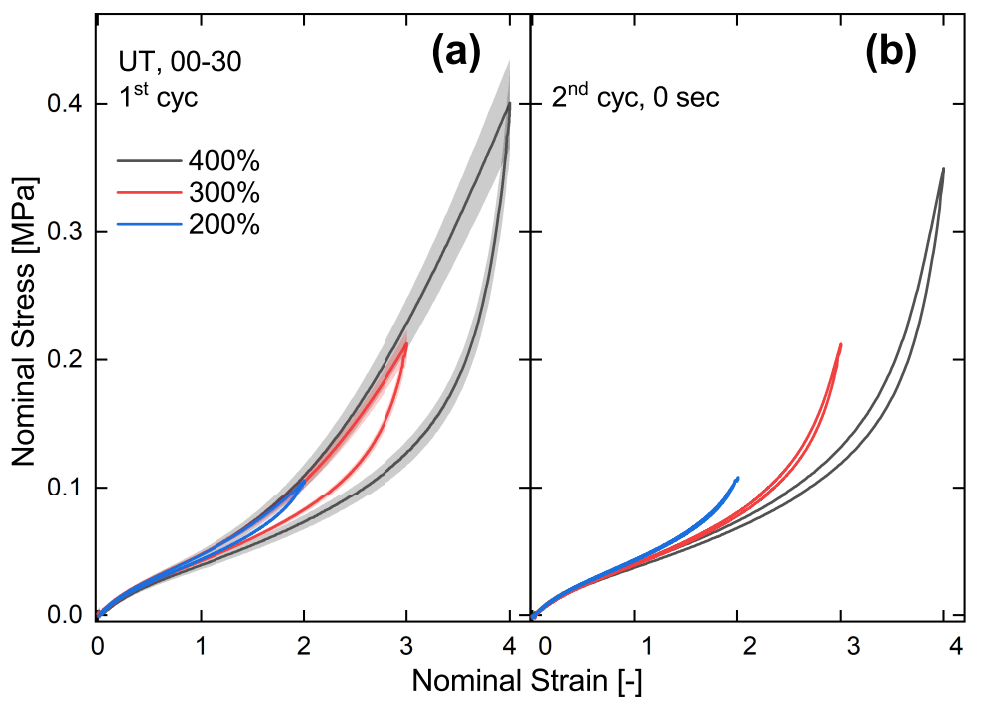

Figure 4. Stress-strain responses of the two-cycle uniaxial tension tests for Shore hardness 00-30 at three strains of 200\%, 300\%, and 400\%. (a) The first cycles with error bands, (b) the second cycles applied 0 second after the first cycle.

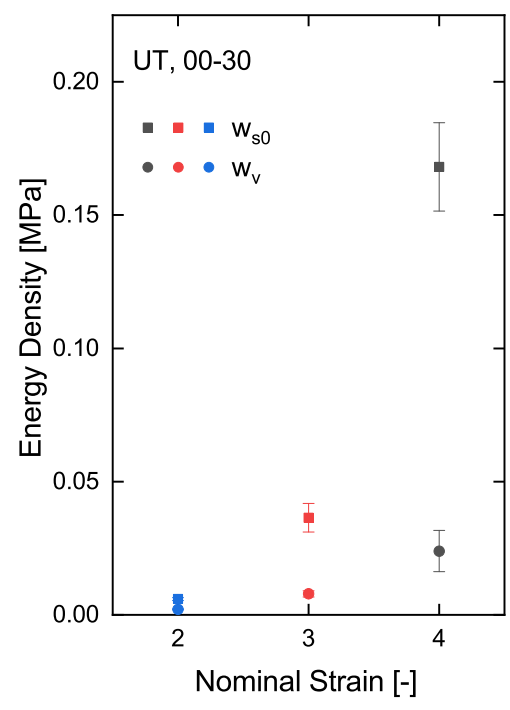

Figure 5. The initial softening dissipation energy density ( $w_{s 0}$, square dots) and viscoelastic dissipation energy density $\left(w_{v}\right.$, circular dots) of uniaxial tension tests for Shore hardness 00-30 at different strains.

\subsection{Uniaxial tension tests}

As a part of observing the stress recovery of Ecoflex polymer, first loading-unloading cycles (denoted as $1^{\text {st }}$ cyc) are applied to virgin specimens to trigger the stress softening. Figure 4(a) depicts the first cycles of uniaxial tensile tests for Ecoflex 00-30 at strains of 200\%, 300\%, and 400\%. The solid lines are the mean values of the first cycles at different strains, while the coloured error bands stand for the standard deviations. Note that the stress softening is found negligible at strain levels below $200 \%$, possibly because the energy threshold of the filler-chain degradation is not met at this stage. Therefore, such a strain range is not included in our experiments. Right after the first cycles, as is mentioned previously, the softening is evacuated with only the minimum recovery. The viscoelastic response can be decoupled by applying the second cycles at the same strain level immediately (denoted as $2^{\text {nd }}$ cyc, 0 sec), see Figure 4(b). Then the initial softening dissipation energy density $w_{s 0}=w_{0}-w_{v}$ is calculated and plotted together with $w_{v}$ in Figure 5 with error bars. It is shown that even though both $w_{s 0}$ and $w_{v}$ increase with strain level, $w_{s 0}$ is markedly larger than $w_{v}$.

After the first cycle, apart from those reloaded immediately in Figure 4(b), all specimens are put to rest at the stress-free state for a certain recovery time before the second cycles to allow for the stress recovery. The second cycles are applied to the specimens under the same experimental conditions as the first cycles. Each subfigure of Figure 6 shows that the stress response for Shore hardness 00-30 is dependent on the recovery time at a certain strain level. Note that for a clear presentation, only the first loading and the second loadings of three different recovery time ( 0 second, 1 day, and 1 month) are presented, and the unloading paths are not shown. At all three strain levels, the stress response of the second loading paths increase and approach to the first loading path with the increase of the recovery times, showing that the subsequent stress response is recovering. As mentioned above, after the softening occurs, some of the polymer chains and fillers are debonded. At the stress-free state after unloading, the molecular thermal motion offers a certain probability to surpass the activation energy for bond rebuilding. Statistically, the probability can be accumulated with 


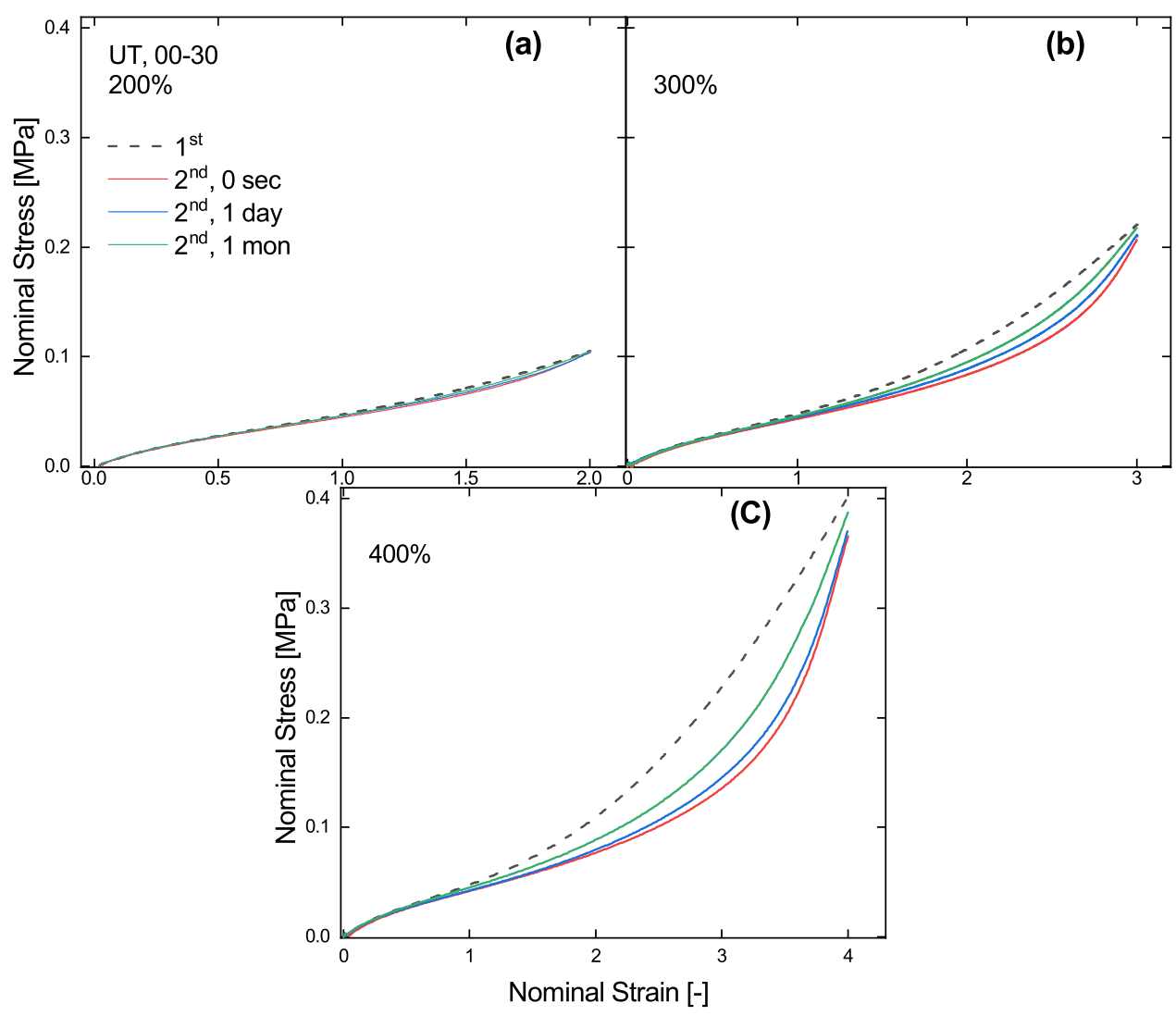

Figure 6. The time dependence of the stress recovery under a uniaxial tension mode for Shore hardness 00-30 at different strains. The second loading paths with recovery times of 0 second, 1 day, and 1 month (solid lines) compared with the first loading path (dash line) at strains of (a) 200\%, (b) 300\%, and (c) 400\%, are presented, respectively.

time. In other words, a longer time results in a greater probability of bond rebuilding, which indicates the macroscopic stress recovery in Figure 6.

To have a quantitative understanding of the stress recovery, the subsequent softening dissipation energy densities $w_{s}$ with different recovery times $t_{r}$ are calculated, see Figure 7(a). Note that all figures with a time axis are plotted in a logarithmic form in this manuscript. It can be seen that as time evolves, the subsequent softening dissipation also increases as a result of the stress recovery. Then the degree of recovery can be evaluated by the ratio of the subsequent dissipation energy density to the initial one $\left(w_{s} / w_{s 0}\right.$, termed as softening dissipation ratio) as is presented in Figure 7(b). At all strain levels, as the recovery time increases, the ratio increases continuously and have a trend to approach $100 \% w_{s} / w_{s 0}$, a full recovery. Considering a certain recovery time with a higher strain level, even though the material generates a larger $w_{s}$ as is shown in Figure 7(a). Regarding the microscopic behaviour, a larger external work is required to trigger the degradation of the filler-rubber interface at a larger strain. This results in more debonded chains and fillers that tend to rebuild bonds at the free-stress state after the unloading. Therefore, more recovery of the absolute softening dissipation is observed in Figure 7(a). However, the recovery is slower as the softening dissipation ratio $w_{s} / w_{s 0}$ is lower than that at a smaller strain level as is shown in Figure 7(b). According to [68], the fillers are not distributed homogeneously but in clusters, leading to the variation of crosslinking densities around the fillers. A filler in the region of higher crosslinking density stands for a tougher filler-rubber link and a 
higher energy threshold of filler-rubber dissociation, which means the dissociation takes place at a higher applied strain than a filler in the region of lower crosslinking density. Correspondingly, the reconnection of such filler-rubber links is tougher. It requires either a longer time for an accumulation of statistical probability of reconnection, which means a slower recovery rate, or a higher temperature to raise the energy to reach the threshold and accelerate the recovery. This phenomenon also reveals that the recovery is retarded at high applied strains [47].

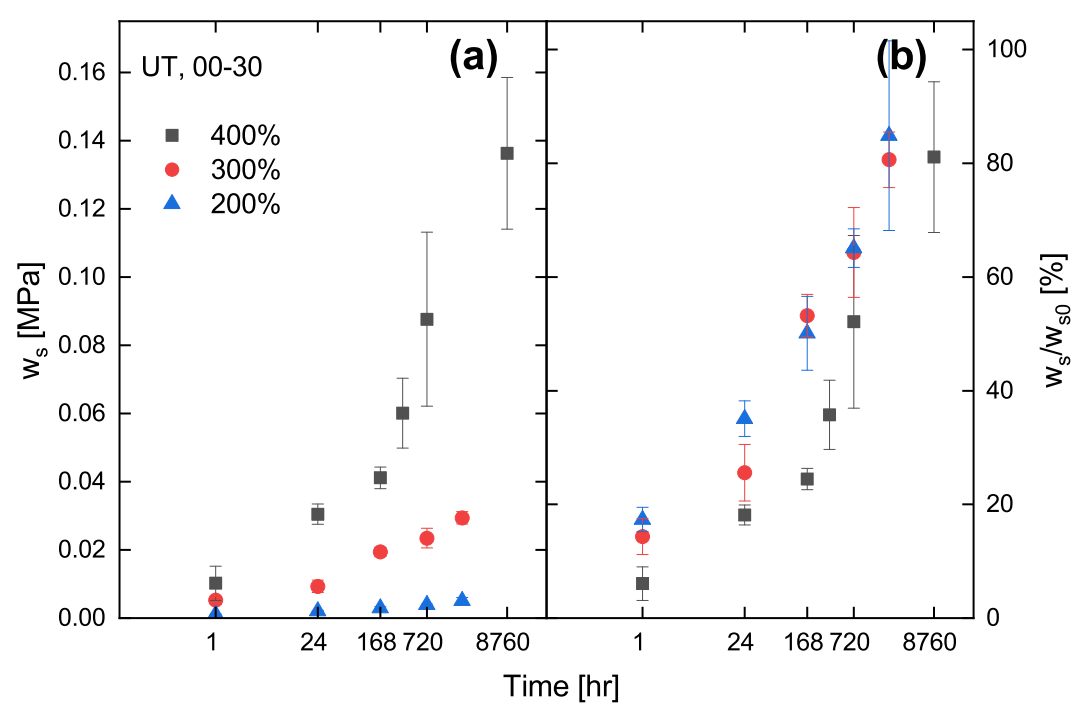

Figure 7. The time dependence of the dissipation during the second cycles of uniaxial tension tests for Shore hardness $00-30$ at strains of $200 \%, 300 \%$, and $400 \%$. (a) Subsequent softening dissipation energy density $\left(w_{s}\right)$, (b) softening dissipation ratio between the subsequent and initial softening dissipation energy density $\left(w_{s} / w_{s 0}\right)$.

\subsection{Planar tension tests}

In view of another deformation mode, planar tension tests are then performed in addition to uniaxial tension. Similar to the uniaxial tension case, first loading-unloading cycles are also applied to virgin specimens to trigger stress softening under planar tension mode, see Figure 8(a). The largest strain level we conducted here is up to $320 \%$. Beyond this strain level, specimens could experience slippery near the grippers. Note that the strains in the figures presented herein are captured by the DIC technique. The viscoelasticity is also decoupled by reloading the second cycle of some specimens 0 second after the first loading, see Figure 8(b). Both the initial softening dissipation $w_{s 0}$ and the viscoelastic dissipation $w_{v}$ under the planar tension mode are illustrated in Figure 9 increase with a higher strain level, sharing similarity with the results of the uniaxial tension case.

The second cycle is also reloaded on each specimen with different recovery periods, showing that the stress level of the second loading path also recovers with a longer recovery time at different strains as shown in Figure 10. Figure 11 quantifies the stress recovery presented in Figure 10 by calculating the subsequent softening dissipation. A higher strain under a planar tension mode gives rise to a larger absolute subsequent softening dissipation energy density $w_{s}$ but a smaller softening dissipation ratio $w_{s} / w_{s 0}$. This is consistent with the results obtained from the uniaxial tension case that the stress recovery is faster at smaller strains. 


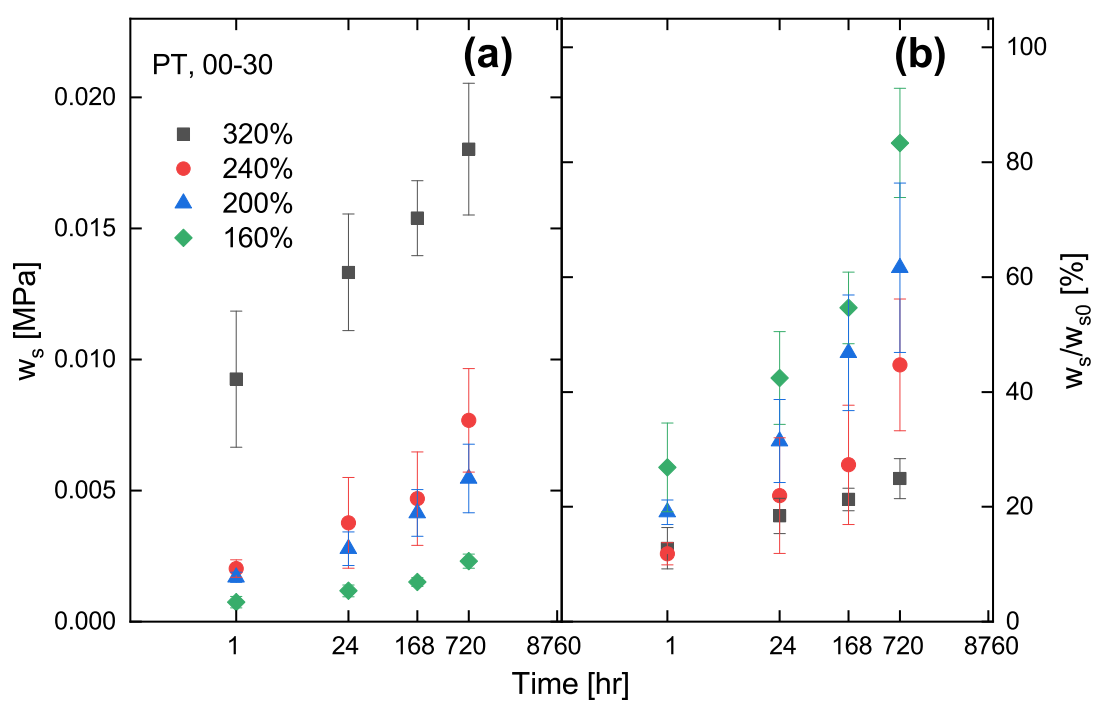

Figure 11. The time dependence of the dissipation during the second cycles of planar tension tests for Shore hardness 00-30 at strains of $160 \%, 200 \%, 240 \%$, and 320\%. (a) Softening dissipation energy density $\left(w_{s}\right)$, (b) softening dissipation ratio between the subsequent and initial softening dissipation energy density $\left(w_{s} / w_{s 0}\right)$.

\subsection{Equibiaxial tension tests}

Additional to the uniaxial tension and planar tension modes, the recovery behaviour under equibiaxial tension mode is also investigated. Here, we only consider the two-cycle loading-unloading equibiaxial tension tests at the strain of $200 \%$ for Shore hardness 00-30. Note that we did not carry out tests over $200 \%$ strains, otherwise the equibiaxial specimens tend to break. For other Shore hardnesses and strain levels, similar recovery behaviour to the uniaxial and planar tension ones can be anticipated. Figure 12 shows the first cycle

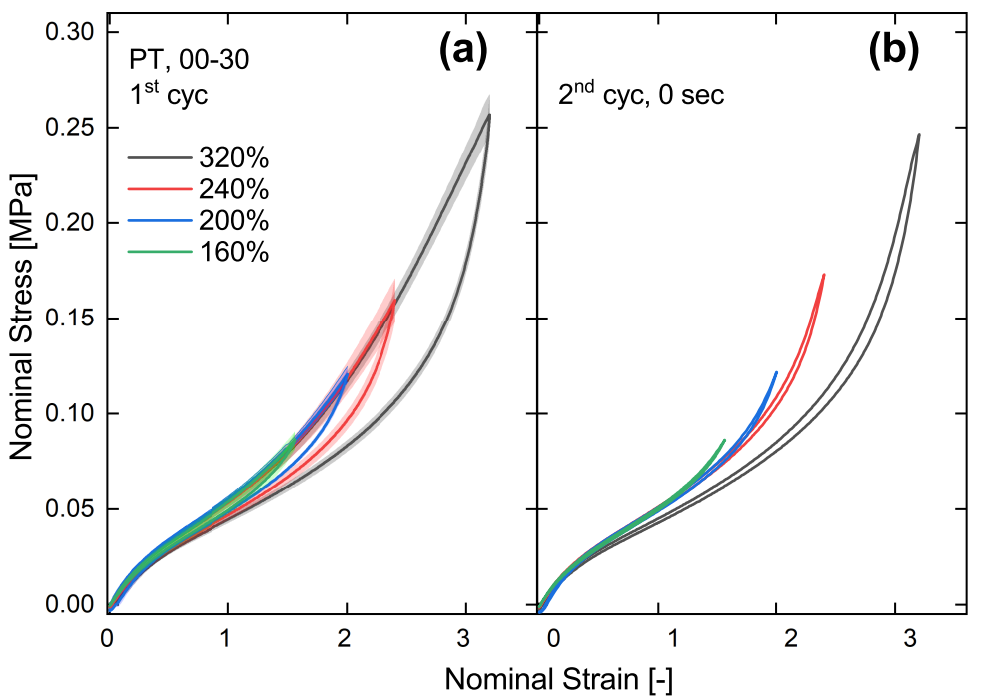

Figure 8. Stress-strain responses of the two-cycle planar tension tests for Shore hardness 00-30 at three strains of $160 \%, 200 \%, 240 \%$, and $320 \%$. (a) The first cycles with error bands, (b) the second cycles applied 0 second after the first cycles.

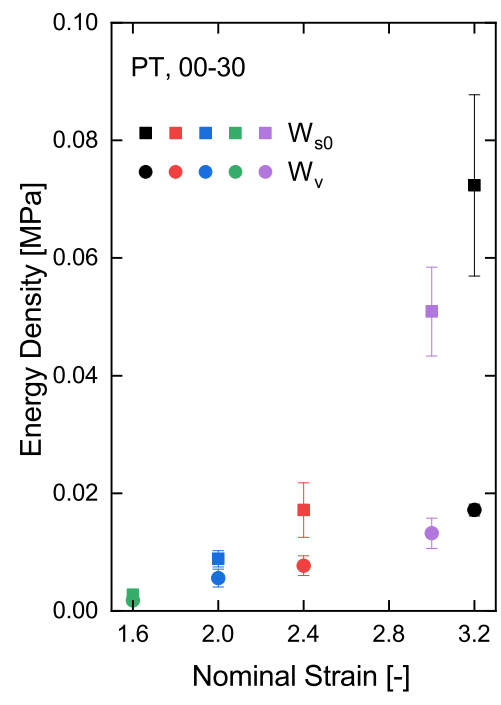

Figure 9. The initial softening dissipation energy density ( $w_{s 0}$, black dots) and viscoelastic dissipation energy density $\left(w_{v}\right.$, red dots) of a planar tension tests for Shore hardness 00-30 at different strains. 


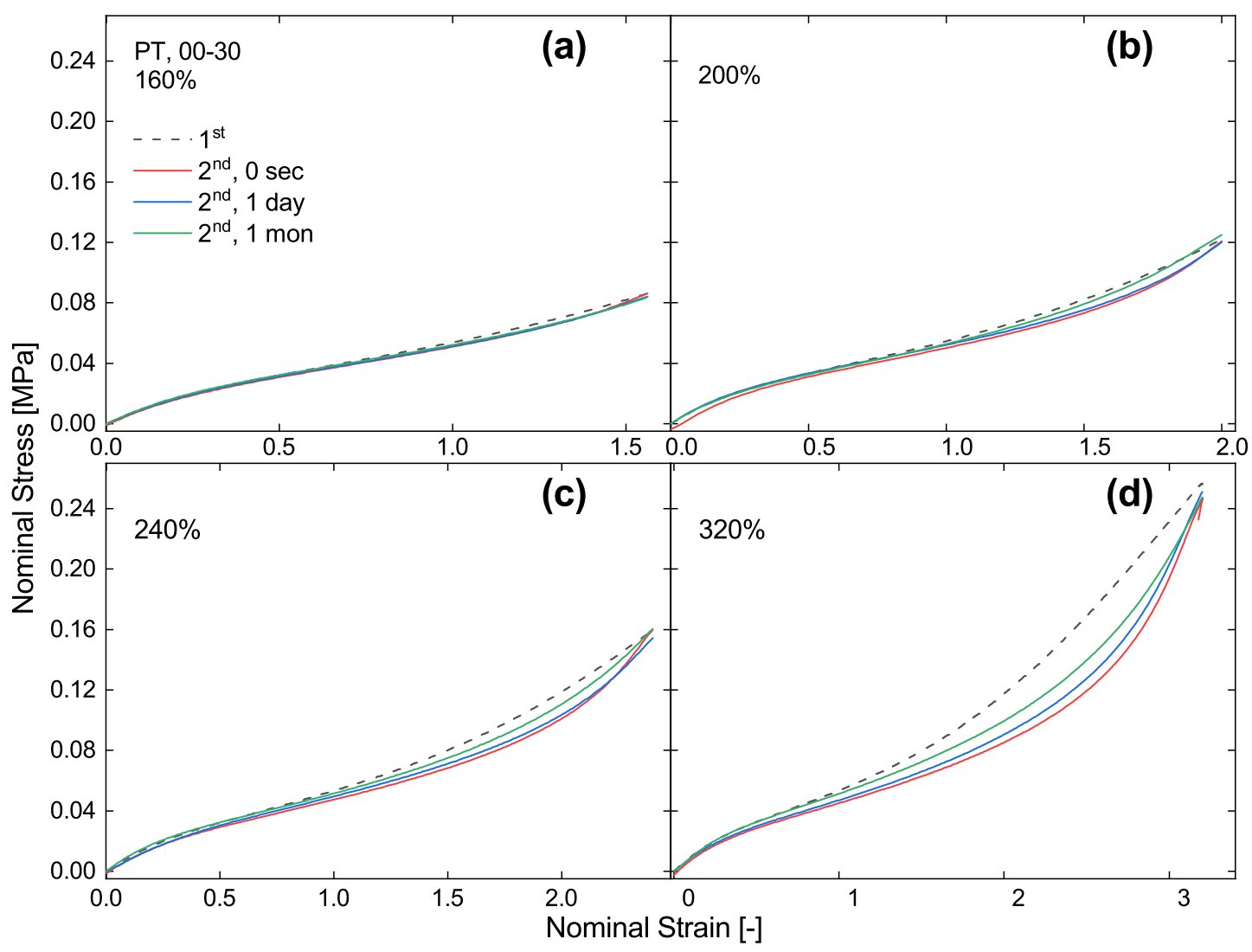

Figure 10. Time dependence of the stress recovery under a planar tension mode for Shore hardness 00-30 at different strains. The second loading paths with recovery times of 0 second, 1 day, and 1 month (solid lines) compared with the first loading path (dash line) at strains of (a) $160 \%$, (b) $200 \%$, (c) $240 \%$, and (d) $320 \%$, are presented, respectively.

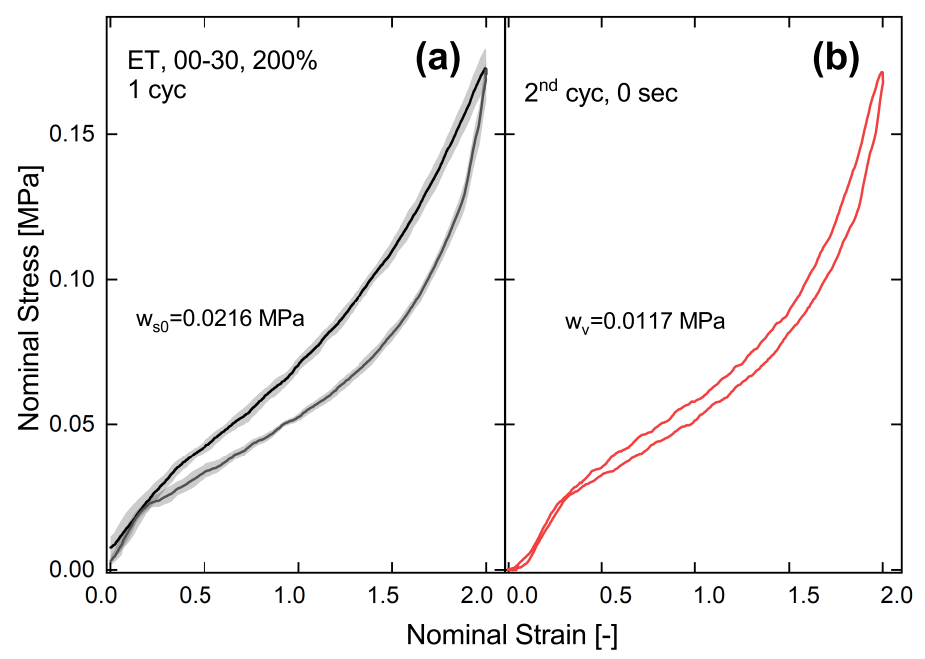

Figure 12. Stress-strain responses of the two-cycle equibiaxial tension tests for Shore hardness 00-30 at three strains of 200\%. (a) The first cycles (black line) with error band, (b) the second cycles applied 0 second after the first cycles (red line). 
and the second cycle reloaded immediately. Accordingly, we obtain the data that the viscoelastic dissipation energy density $w_{v}$ equals $0.0117 \pm 0.0032 \mathrm{MPa}$ while the initial softening dissipation energy density $w_{s} 0$ equals $0.0215 \pm 0.0045 \mathrm{MPa}$. The stress-strain of the second reloading at different reloading time and the corresponding subsequent softening dissipation energy density $w_{s 0}$ is presented in Figure 13 and Figure 14, respectively. These figures confirm that the stress response and softening dissipation recover with time, revealing that the stress recovery also exists under the equibiaxial tension mode.

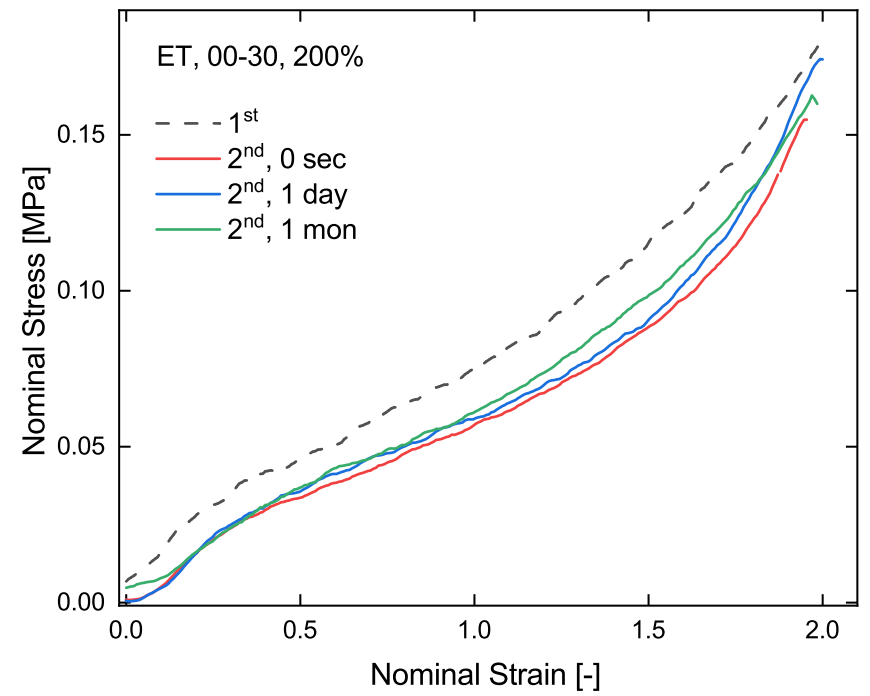

Figure 13. Time dependence of the stress recovery under an equibiaxial tension mode for Shore hardness 00-30 at strains of 200\%. The second loading paths with recovery times of 0 second, 1 day, and 1 month (solid lines) compared with the first loading path (dash line) are presented.

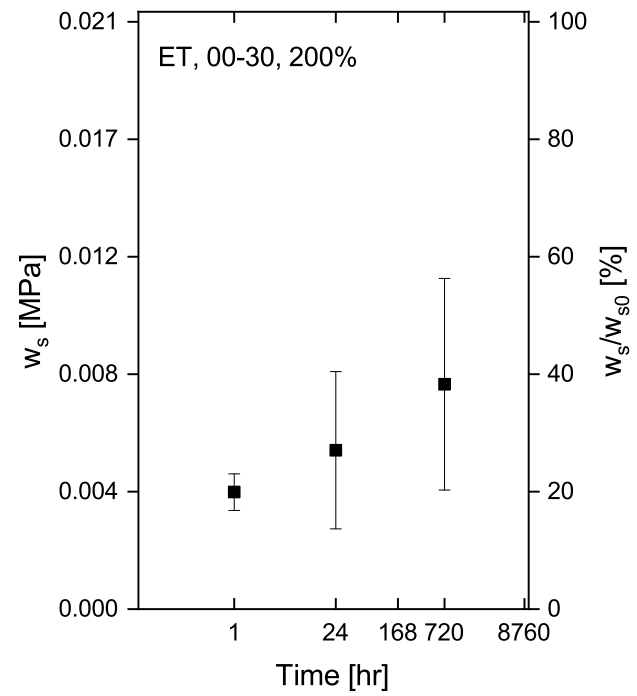

Figure 14. Time dependence of the softening dissipation during the second cycles of equibiaxial tension tests for Shore hardness 00-30 at strains of 200\%. The left $\mathrm{Y}$ axis: subsequent softening dissipation energy density $\left(w_{s}\right)$, and the right $\mathrm{Y}$ axis: the softening dissipation ratio between the subsequent and initial softening dissipation energy density $\left(w_{s} / w_{s 0}\right)$

\subsection{Comparisons of the stress recoveries for the different deformation modes}

In previous sections, we discussed experimental results performed under a certain deformation mode separately. In this section, data under three different deformation modes, i.e., uniaxial tension, planar tension and equibiaxial tension at the strain of 200\% for Shore hardness 00-30 are compared, including the stressstrain response of the first cycles and immediately reloaded second cycles (see Figure 15(a) and (b)), initial softening dissipation $w_{s 0}$ and viscoelastic dissipation $w_{v}$ increase (see Figure 16), and the subsequent softening dissipation $w_{s}$ and the corresponding softening dissipation ratio $w_{s} / w_{s 0}$ (see Figure 17(a) and (b)). From Figure 15 and Figure 16, we can find clearly that the stress level and both the initial softening and the viscoelastic dissipation increase with the increase of the biaxiality ratio $\mu$. It is noteworthy to mention that under the uniaxial tension mode, where $\mu=-1 / 2$, the second loading and unloading paths reloaded immediately after the first cycles are almost overlapped along the first unloading path. That means only negligible viscoelastic dissipation $w_{v}$ is produced, and most parts of the dissipation in the first cycle are the contribution of the stress softening. In comparison, when $\mu=0$, i.e., under planar tension mode and $\mu=1$, i.e., under equibiaxial tension mode, the second unloading goes along a distinct path against the second loading path, and the viscoelastic dissipation $w_{v}$ takes up a large part of the total dissipation that cannot be ignored. 


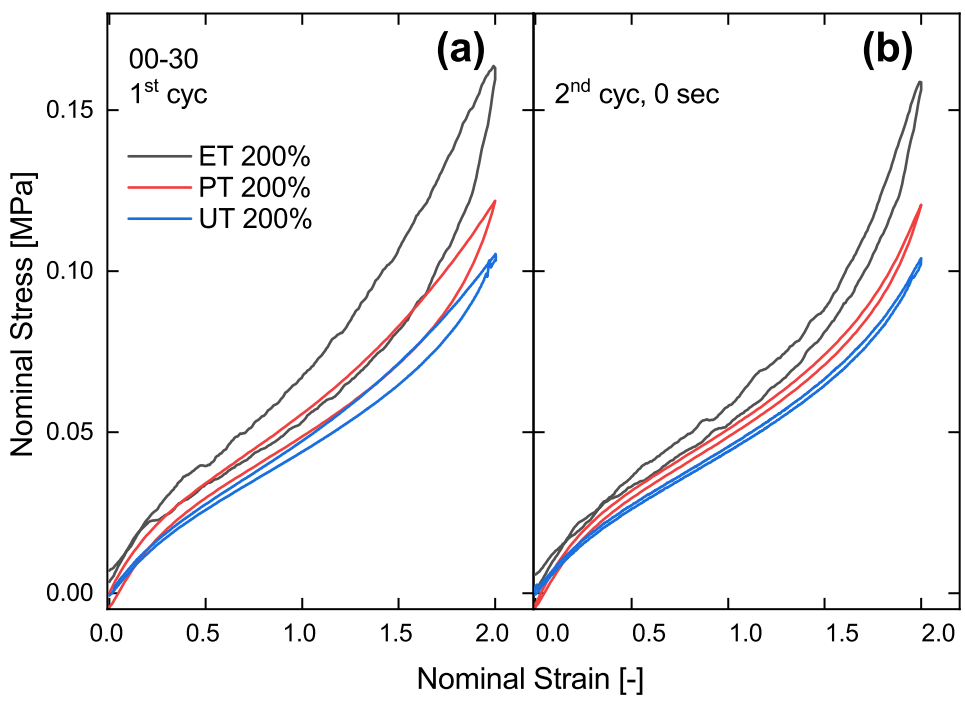

Figure 15. Stress-strain responses of the two-cycle uniaxial tension tests for Shore hardness 00-30 at strains of 200\% under deformation mode of uniaxial tension, planar tension and equibiaxial tension. (a) The first cycles, (b) the second cycles applied 0 second after the first cycles.

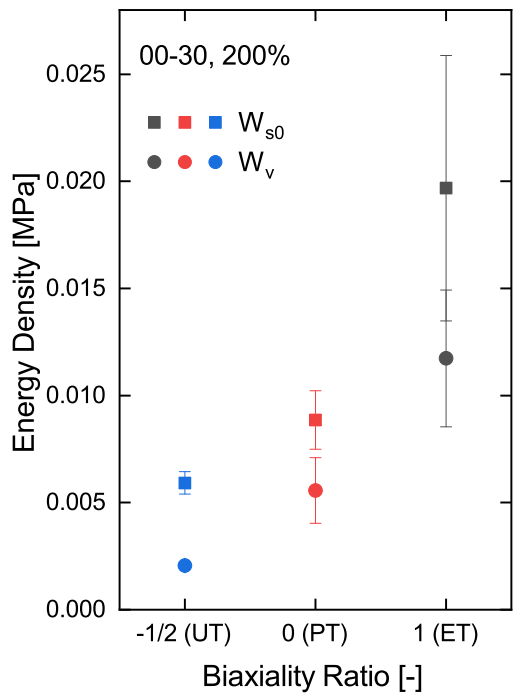

Figure 16. The initial softening dissipation energy density ( $w_{s 0}$, black dots) and viscoelastic dissipation energy density $\left(w_{v}\right.$, red dots) for Shore hardness 00-30 at strains of $200 \%$ under different deformation mode (biaxiality ratio).

From a microscopic perspective, due to the chain-chain interactions, the stretch of polymer in the transverse direction leads to the tendency of reorientation and extra deformation for chains in other directions. Therefore, with a larger $\mu$, the axial polymer chains are extended more, and the stress responses including stress softening and stress recovery at a certain axial strain resemble those at a larger equivalent uniaxial strain, for example, the resemblance between Figure 7 and Figure 17.

\subsection{Shore hardness dependence}

In addition to the strain-dependence, the Shore hardness of the materials could also affect the stress recovery. Figure 18(a) illustrates the first cycles of virgin specimens for different Shore hardnesses, and Figure 18(b) illustrates the second cycles applied 0 second after the first loading. At both cycles, the stress level is higher for a higher Shore hardness. With the data of first cycles and second cycles after 0 second, the initial softening dissipation energy density $w_{s 0}$ and the viscoelastic dissipation energy density $w_{v}$ can also be decoupled and obtained, as Figure 19 shows. Again, $w_{s 0}$ is significantly larger than $w_{v}$. Except that both dissipations of Shore hardness 00-10 are slightly higher than those of 00-20, overall a higher Shore hardness yields a larger $w_{s 0}$ and $w_{v}$. Note that according to the product information of Ecoflex series, the viscosity for 00-10 


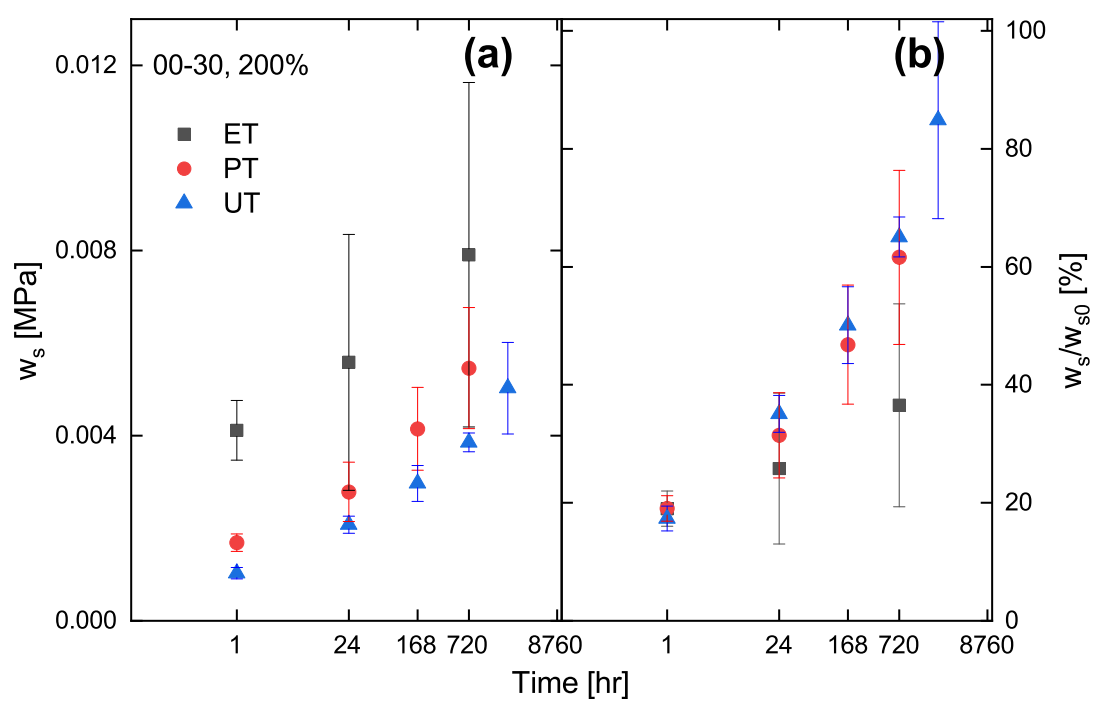

Figure 17. Time dependence of the dissipation during the second cycles at strains of $200 \%$ for Shore hardness 00-30 under deformation modes of uniaxial tension, planar tension, and equibiaxial tension. (a) The subsequent softening dissipation energy density $\left(w_{s}\right)$, (b) the softening dissipation ratio between the subsequent and initial softening dissipation energy density $\left(w_{s} / w_{s 0}\right)$.

is higher than 00-20.

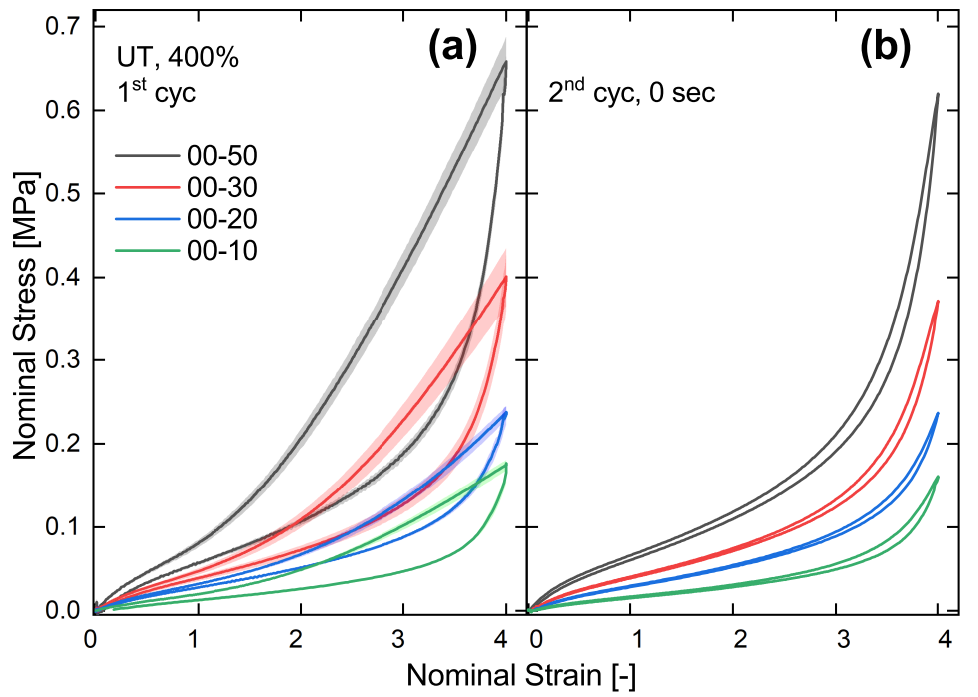

Figure 18. Stress-strain response of the two-cycle uniaxial tension tests at strains of $400 \%$ for four Shore hardnesses 00-10, 00-20, 00-30, 00-50. (a) The first cycles with error bands, (b) the second cycles applied 0 second after the first cycle.

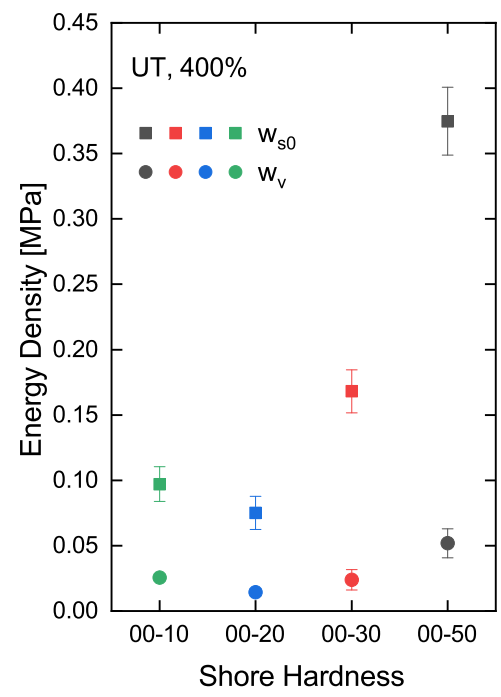

Figure 19. The initial softening dissipation energy density ( $w_{s 0}$, black dots) and viscoelastic dissipation energy density $\left(w_{v}\right.$, red dots) of uniaxial tension tests at strains of $400 \%$ for different Shore hardnesses.

After different recovery times under the stress-free state, the second cycles are applied under the same conditions as their first cycles for all four Shore hardnesses. Each subfigure of Figure 20 depicts the stress-strain curves of the first loading and the second loadings of three recovery times (again, 0 second, 1 day and 1 


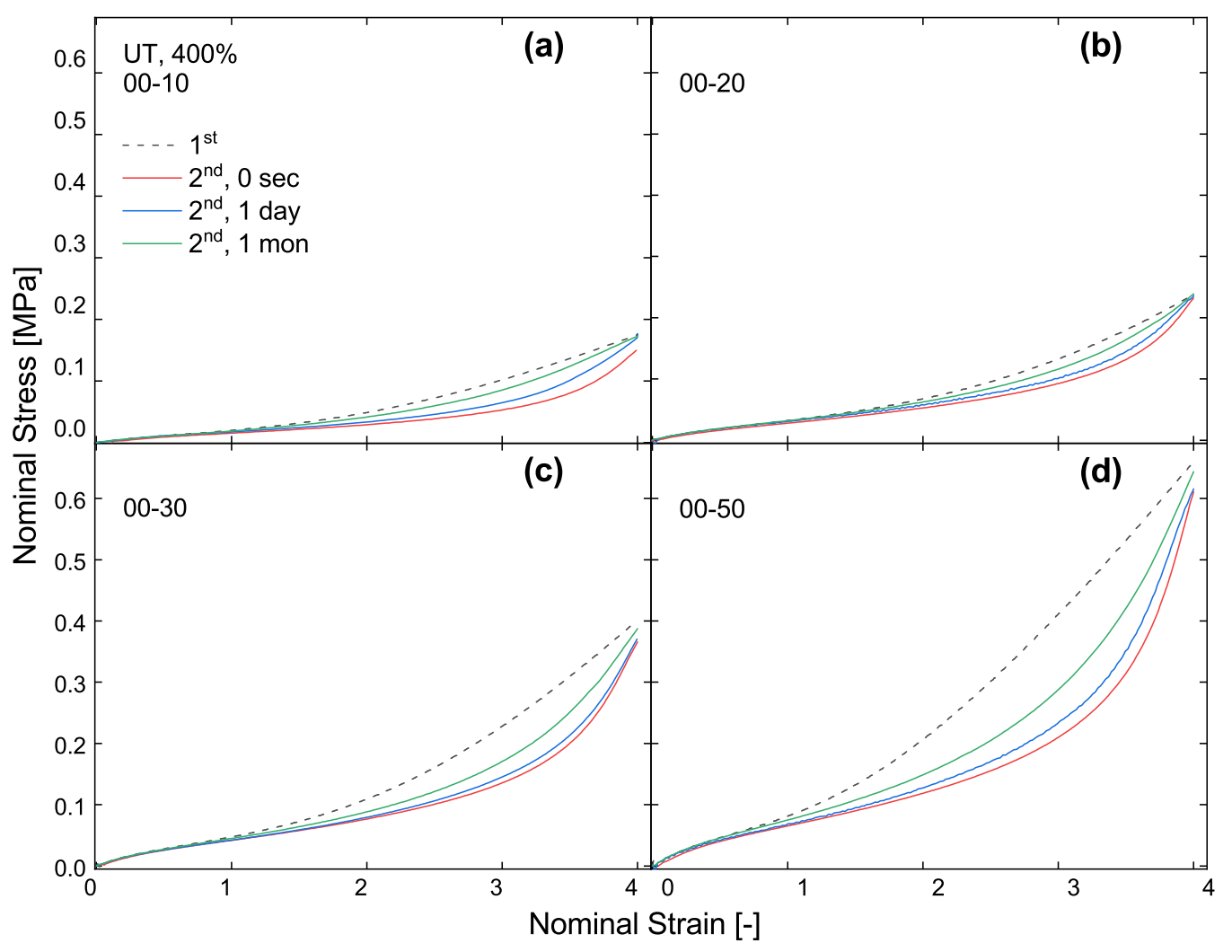

Figure 20. Time dependence of the stress recovery under a uniaxial tension mode at strains of $400 \%$ for different Shore hardnesses. The second loading paths with recovery times of 0 second, 1 day, and 1 month (solid lines) compared with the first loading path (dash line) for Shore hardnesses (a) 00-10, (b) 00-20, (c) 00-30, (d) 00-50, are presented, respectively.

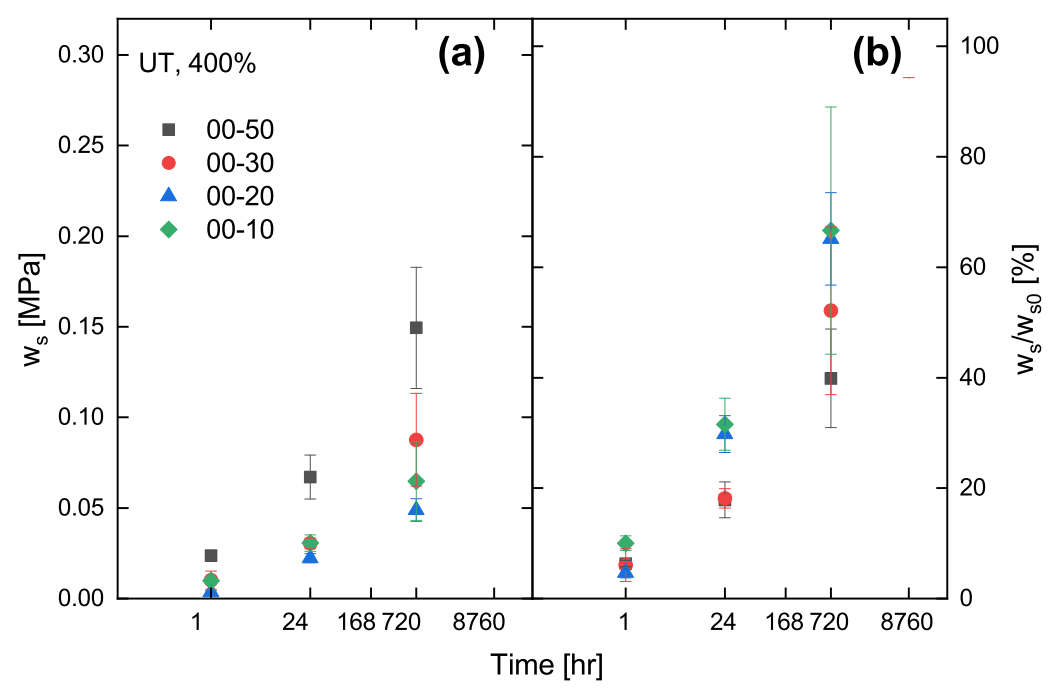

Figure 21. The time dependence of the dissipation during the second cycles of uniaxial tension tests at strains of $400 \%$ for different Shore hardnesses. (a) The subsequent softening dissipation energy density $\left(w_{s}\right)$, (b) the softening dissipation ratio between the subsequent and initial softening dissipation energy density $\left(w_{s} / w_{s 0}\right)$. 
month) for a particular Shore hardness. It demonstrates clearly that the stress responses for all four hardnesses recover with time. With regard to the softening dissipation energy density, as is shown in Figure 21, a larger Shore hardness has a higher subsequent softening dissipation energy density $w_{s}$ except for Shore 00-10. It is also highlighted in the technical manual that the viscosity of 00-10 is significantly larger than other Shore hardness in the series. However, the softening dissipation ratio $w_{s} / w_{s 0}$ is in an opposite trend, i.e., a larger Shore hardness recovers at a slower speed than the smaller one regarding a certain recovery time for all four Shore hardnesses.

Under planar tension, the dependence of Shore hardness is also observed. Figure 23 illustrates that a larger Shore hardness has a higher absolute softening dissipation $w_{s}$, while a larger Shore hardness yields a lower softening dissipation ratio $w_{s} / w_{s 0}$ at a certain recovery time, showing a very similar pattern as the uniaxial one in Figure 21.

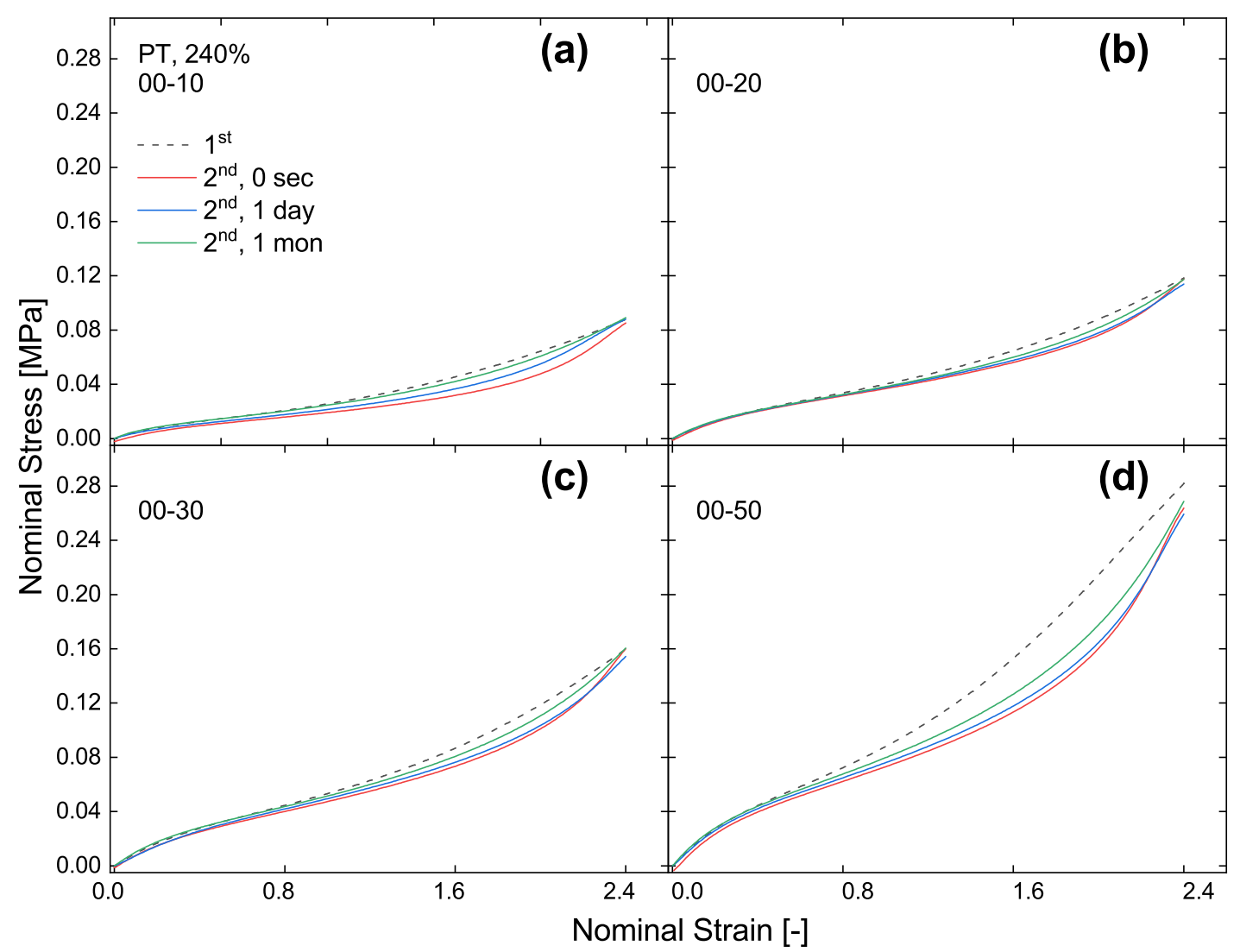

Figure 22. Time dependence of the stress recovery under a planar tension mode at strains of $240 \%$ for different Shore hardnesses. The second loading paths with recovery times of 0 second, 1 day, and 1 month (solid lines) compared with the first loading paths (dash line) for Shore hardnesses (a) 00-10, (b) 00-20, (c) 00-30, (d) 00-50, are presented, respectively.

With the help of thermogravimetric analysis, Krpovic et al. [64] obtained that Ecoflex 00-50 contains a larger amount of fillers than 00-30. Besides, using the nuclear magnetic resonance spectroscopy and Fourier transform infrared spectroscopy analyses, they found that Ecoflex 00-50 has a higher crosslinking degree than 00-30. With the increase of filler volume fraction, the polymer matrix forms more physically crosslinked networks. Therefore, both the crosslinking density and the filler volume fraction contribute to the total re- 


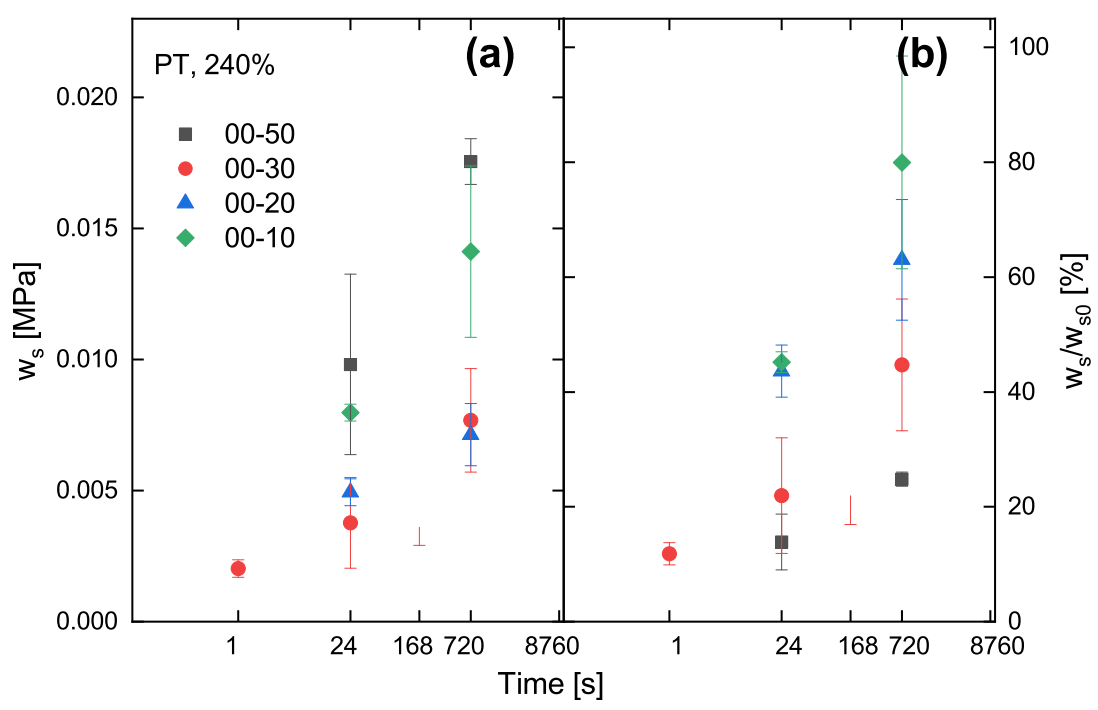

Figure 23. The time dependence of the dissipation during the second cycles of planar tension tests at strains of $240 \%$ for Shore hardnesses 00-10, 00-20, 00-30, 00-50. (a) The subsequent softening dissipation energy density $\left(w_{s}\right)$, (b) the softening dissipation ratio between the subsequent and initial softening dissipation energy density $\left(w_{s} / w_{s 0}\right)$.

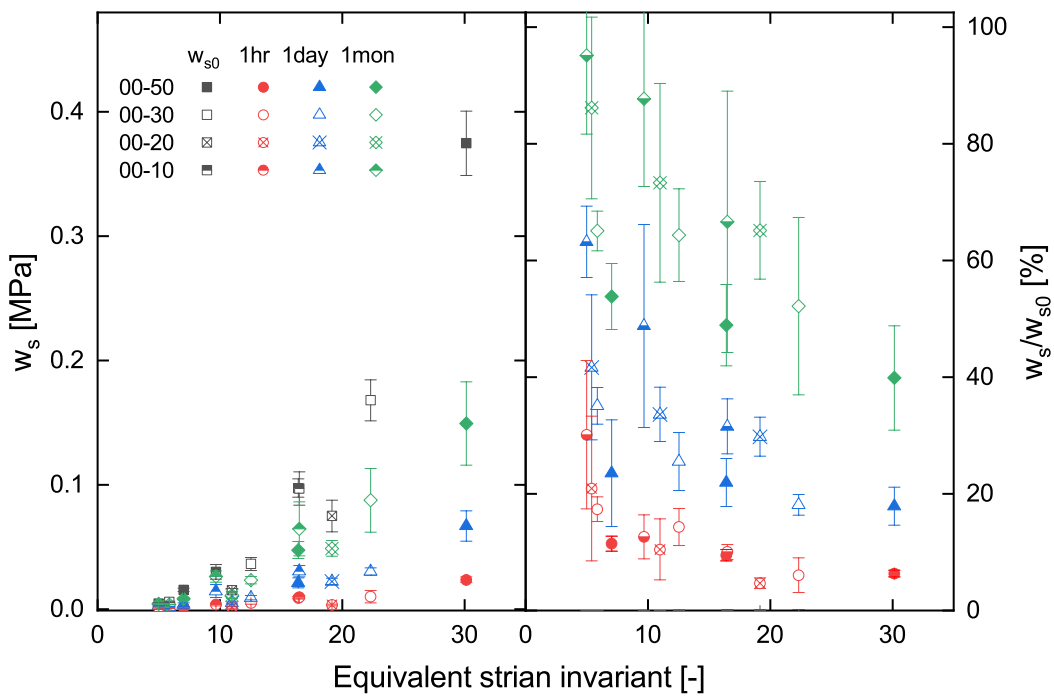

Figure 24. The softening energy density for different Shore hardness with the equivalent strain invariant. (a) The subsequent softening dissipation energy density $\left(w_{s}\right)$, (b) the softening dissipation ratio between the subsequent and initial softening dissipation energy density $\left(w_{s} / w_{s 0}\right)$.

inforcement of the materials. Accordingly, a higher Shore hardness results in a stronger stiffness as well as a greater stress softening during the loading-unloading cycle. As we mentioned in Section 3.2, a higher crosslinking density leads to a more significant softening as well as a slower recovery rate. Now, a larger Shore hardness of Ecoflex has a larger filler volume fraction and a higher crosslinking density which makes it plausible that a slower recovery rate for a higher Shore hardness can be observed as in Figure 21.

In previous sections, we mentioned that the crosslinking degree determines the entropic elasticity of the 
polymer networks, while the fillers cause the stress softening. According to literature [46, 47], the fillers are supposed to cause deformation concentration in the polymer network, and therefore the microscopic deformation is larger than the macroscopic one. In other words, a higher filler volume can be regarded as amplification of the strain. This phenomenon is characterised by introducing a strain amplification factor for the uniaxial stretch [69] or amplifying the first strain invariant $I_{1}$ in the case of a three-dimensional deformation [70], and this concept is adopted frequently, see [71, 72, 73, 74]. Li et al. [47] used the strain amplification factor to investigate the viscoelastic response, stress softening as well as the stress recovery. Note that in our previous work [38], we generalised the strain amplification factor for the case of Shore hardness variation regardless of the unknown commercial formulation of Ecoflex, and successfully modelled the stress-strain response and stress softening behaviour for different Shore hardnesses. We amplified the first strain invariant $I_{1}$ to the equivalent first invariant $I_{1, h}$ as

$$
I_{1, h}=X\left[I_{1}-3\right]+3,
$$

where, the strain amplification factor $X$ is a function of a normalised variable $s$ as

$$
\begin{gathered}
X=1+g_{1} s+g_{2} s^{2}, \\
s=\frac{H}{H_{0}}-1,
\end{gathered}
$$

where, $g_{1}=1.788 \mathrm{e}-01$ and $g_{2}=1.851 \mathrm{e}-02$ are the parameters that were identified in Liao et al. [38]. Note that $H$ is the last two digits of a Shore hardness, and 00-10 is taken as the reference Shore hardness $H_{0}$. Here, the variation $s$ serves similarly to the filler fraction $(\varphi)$ in the literature. Using the superposition method adopted in [47] based on Equation 4 to Equation 6, the softening dissipations at various strain levels $(200 \%, 300 \%$, and $400 \%)$ for all four Shore hardnesses (00-10, 00-20, 00-30, and 00-50) are plotted on the equivalent first invariant $I_{1, h}$ axis as Figure 24. Figure 24(a) shows the superposed initial softening dissipation $w_{s 0}$ and subsequent softening dissipation $w_{s}\left(t_{r}\right)$ including $t_{r}=1 \mathrm{hr}, 1$ day, 1 month. It reveals that the stress softening and the stress recovery are dependent on the amplified microscopic deformation. We can see at each recovery time, the data basically falls in one monotonically increasing line with the increase of $I_{1, h}$. Comparing data at different recovery times, the subsequent dissipation is regaining towards the initial softening dissipation. In light of $w_{s} / w_{s 0}$ against $I_{1, h}$, it shows an opposite trend that a larger equivalent first invariant $I_{1, h}$ the recovery is slower, indicating the retardation of rebuilding the chain-filler interface as mentioned previously. Overall, the results again confirm the effectiveness of the strain amplification factor for the Shore hardness.

\section{Conclusions}

In this study, we have carried out a series of systematic experimental studies on Ecoflex, a widely-used silicone rubber in soft robotics, stretch-based sensors, and energy harvesters. In this regard, stress softening and stress recovery behaviour have extensively been studied with respect to recovery time, strain level, deformation modes, and Shore hardness. Our findings can be summarised as follows:

- The total energy dissipation of a loading-unloading cyclic test could be decomposed into viscoelastic dissipation and softening dissipation. The stress softening is triggered on the first cycle, and stress recovery commences at the stress-free state with time and can be observed on the subsequent cycles, 
- A larger strain level results in more significantly stress softening but slower stress recovery with regard to the softening dissipation ratio,

- A larger biaxiality ratio of the deformation mode also causes more apparent stress softening as well as a slower stress recovery.

- The Shore hardness dependence of the stress recovery shares a similarity to the strain level dependence. The variation of the Shore hardness can be regarded as the strain amplification. Using the strain amplification factor, the Shore hardness dependence can be confirmed and is consistent with our previous work [38].

From a microscopic point of view, the stress softening is strongly dependent on filler and matrix (rubber) interactions. In fact, the strain level, biaxiality ratio, and the Shore hardness dependences are all able to be interpreted as the dependence of microscopic deformation of polymer chains. Overall, a microscopic larger stretch causes more debonding of the rubber matrix and the fillers, and therefore more energy and longer time are required for the bond rebuilding of the fillers and the rubber matrix. Therefore, it takes a longer recovery time for the material to recovery to the same level of softening dissipation ratio. This work gives a comprehensive investigation of the factors that influence the stress recovery process and reveals that the microscopic reasons for the dependence of these factors coincide. Since Ecoflex silicone rubbers and other congeneric polymers often experience multiple complex deformations, their stress softening and recovery are of great importance to predict the long-term mechanical responses of a product made of such soft polymeric materials. Our experimental data shed light on modelling the complete constitutive mechanical response as depicted in Figure 3 including hyperelasticity, viscoelasticity, stress softening, and stress recovery.

\section{Appendix A The application of the DIC technique}

In order to confirm further the homogeneity of the specimen deformation, the DIC technique is adopted. Figure 25 shows the contour of the axial strain filed of a specimen for the planar tension. It can be seen the strain filed is homogeneous in the main part. In addition, Figure 26 shows the transverse strain field of the same deformed specimen. The overall value is close to zero in most part of the specimen. Therefore, the assumption of negligible in-plane transverse deformation is satisfied. The DIC technique is also utilised to analyse the test for equibiaxial tension. Figure 27 shows the relatively homogeneous strain contour of an equibiaxial tension specimen in the central region.

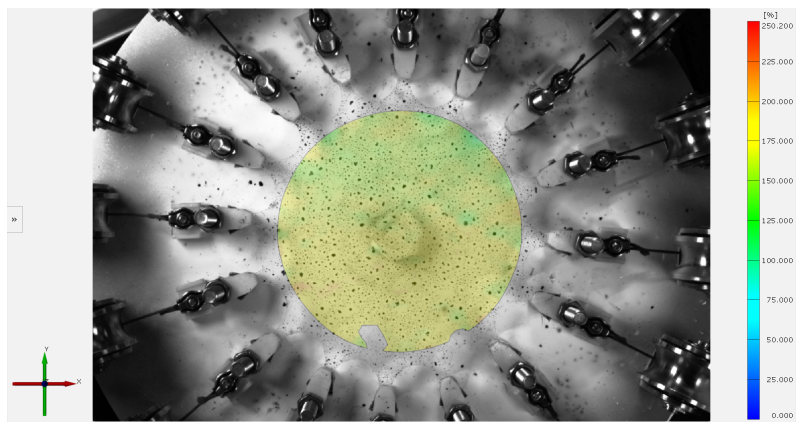

Figure 27. The DIC countour of strain of a equibiaxial test test. 


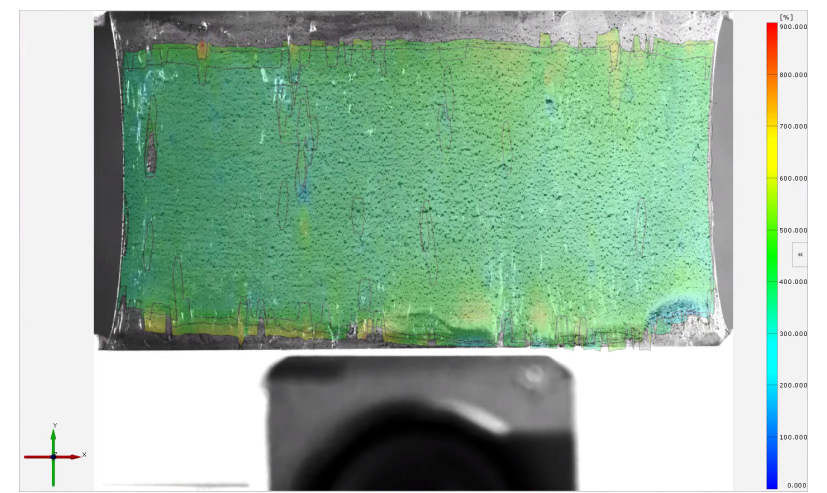

Figure 25. The DIC Contour of axial deformation of a planar tension test.

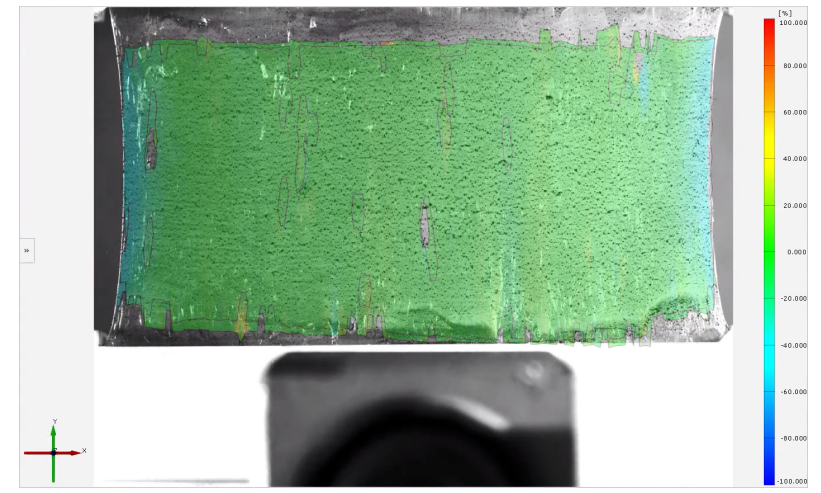

Figure 26. The DIC Contour of width transverse deformation.

\section{Acknowledgements}

This work is partially supported by the National Science Fund for Distinguished Young Scholar (No. 11925203), the National Natural Science Foundation of China (No. 1672110), and the Open Project Program of State Key Laboratory of Traction Power under Grant (No. TPL2003). MH also acknowledges the support by EPSRC through the Supergen ORE Hub (EP/S000747/1), who have awarded funding for the Flexible Fund project Submerged bi-axial fatigue analysis for flexible membrane Wave Energy Converters (FF2021-1036).

\section{References}

[1] L. Bernardi, R. Hopf, A. Ferrari, A. E. Ehret, E. Mazza, On the large strain deformation behavior of silicone-based elastomers for biomedical applications, Polymer Testing 58 (2017) 189-198. doi: $10.1016 / j$.polymertesting.2016.12.029.

[2] R. Hopf, L. Bernardi, J. Menze, M. Zündel, E. Mazza, A. E. Ehret, Experimental and theoretical analyses of the age-dependent large-strain behavior of Sylgard 184 (10:1) silicone elastomer, Journal of the Mechanical Behavior of Biomedical Materials 60 (2016) 425-437. doi:10.1016/j.jmb.bm . 2016.02 .022 . 
[3] H. Hoeksema, M. De Vos, J. Verbelen, A. Pirayesh, S. Monstrey, Scar management by means of occlusion and hydration: A comparative study of silicones versus a hydrating gel-cream, Burns 39 (7) (2013) 1437-1448. doi:10.1016/j.burns.2013.03.025.

[4] J. W. Stansbury, M. J. Idacavage, 3D printing with polymers: Challenges among expanding options and opportunities, Dental Materials 32 (1) (2016) 54-64. doi:10.1016/j. dental.2015.09.018.

[5] A. Unkovskiy, S. Spintzyk, J. Brom, F. Huettig, C. Keutel, Direct 3D printing of silicone facial prostheses: A preliminary experience in digital workflow, Journal of Prosthetic Dentistry 120 (2) (2018) 303-308. doi:10.1016/j.prosdent.2017.11.007.

[6] A. D. Lee, P. Shepherd, M. C. Evernden, D. Metcalfe, Measuring the effective Young's modulus of structural silicone sealant in moment-resisting glazing joints, Construction and Building Materials 181 (2018) 510-526. doi:10.1016/j.conbuildmat.2018.06.038.

[7] X. Li, Q. Huang, J. Deng, G. Zhang, Z. Zhong, F. He, Evaluation of lithium battery thermal management using sealant made of boron nitride and silicone, Journal of Power Sources 451 (2020). doi:10.1016/j.jpowsour.2020.227820.

[8] R. Cardoso, A. C. Balestro, A. Dellallibera, E. C. M. Costa, J. M. G. Angelini, L. H. I. Mei, Silicone insulators of power transmission lines with a variable inorganic load concentration: Electrical and physiochemical analyses, Measurement: Journal of the International Measurement Confederation 50 (1) (2014) 63-73. doi:10.1016/j.measurement.2013.12.030.

[9] J. C. Case, E. L. White, R. K. Kramer, Soft material characterization for robotic applications, Soft Robotics 2 (2) (2015) 80-87. doi:10.1089/soro.2015.0002.

[10] M. Cianchetti, C. Laschi, A. Menciassi, P. Dario, Biomedical applications of soft robotics, Nature Reviews Materials 3 (6) (2018) 143-153. doi:10.1038/s41578-018-0022-y.

[11] T. J. Wallin, J. Pikul, R. F. Shepherd, 3D printing of soft robotic systems, Nature Reviews Materials 3 (6) (2018) 84-100. doi:10.1038/s41578-018-0002-2.

[12] V. R. Jayaneththi, K. C. Aw, A. J. McDaid, Coupled magneto-mechanical modeling of non-linear ferromagnetic diaphragm systems, International Journal of Mechanical Sciences 155 (November 2018) (2019) 360-369. doi:10.1016/j.ijmecsci.2019.03.003.

[13] Y. Mei, B. Stover, N. Afsar Kazerooni, A. Srinivasa, M. Hajhashemkhani, M. R. Hematiyan, S. Goenezen, A comparative study of two constitutive models within an inverse approach to determine the spatial stiffness distribution in soft materials, International Journal of Mechanical Sciences 140 (November 2017) (2018) 446-454. doi:10.1016/j.ijmecsci.2018.03.004. URL https://doi.org/10.1016/j.ijmecsci.2018.03.004

[14] A. Kumar, K. Patra, M. Hossain, Silicone composites cured under a high electric field: an electromechanical experimental study, Polymer Composites (2020). doi:10.1002/pc.25875.

[15] M. Amjadi, Y. J. Yoon, I. Park, Ultra-stretchable and skin-mountable strain sensors using carbon nanotubes-Ecoflex nanocomposites, Nanotechnology 26 (37) (2015). doi:10.1088/ $0957-4484 / 26 / 37 / 375501$. 
[16] I. Collins, M. Hossain, W. Dettmer, I. Masters, Flexible membrane structures for wave energy harvesting: A review of the developments, materials, and computational modelling approaches, Renewable and Sustainable energy Reviews (In press) (2021).

[17] C. L. Zhang, Z. H. Lai, X. X. Rao, J. W. Zhang, D. Yurchenko, Energy harvesting from a novel contact-type dielectric elastomer generator, Energy Conversion and Management 205 (2020). doi: $10.1016 / j$.enconman.2019.112351.

[18] M. Mariello, L. Fachechi, F. Guido, M. De Vittorio, Multifunctional sub-100 $\mu$ m thickness flexible piezo/triboelectric hybrid water energy harvester based on biocompatible AlN and soft parylene CPDMS-Ecoflex ${ }^{\mathrm{TM}}$, Nano Energy 83 (2021) 105811. doi:10.1016/j. nanoen.2021.105811. URL https:// linkinghub.elsevier.com/retrieve/pii/s2211285521000690

[19] H. Mai, R. Mutlu, C. Tawk, G. Alici, V. Sencadas, Ultra-stretchable MWCNT-Ecoflex piezoresistive sensors for human motion detection applications, Composites Science and Technology 173 (2019) 118-124. doi:10.1016/j.compscitech.2019.02.001.

[20] Y. Jiang, Y. Wang, Y. K. Mishra, R. Adelung, Y. Yang, Stretchable CNTs-Ecoflex Composite as Variable-Transmittance Skin for Ultrasensitive Strain Sensing, Advanced Materials Technologies 3 (12) (2018). doi:10.1002/admt . 201800248.

[21] L. An, T. Lu, J. Xu, Z. Wang, M. Xu, T. J. Wang, Soft sensor for measuring wind pressure, International Journal of Mechanical Sciences 141 (January) (2018) 386-392. doi:10.1016/j.ijmecsci. 2018.01 .038$.

[22] Z. Xu, S. Zheng, X. Wu, Z. Liu, R. Bao, W. Yang, M. Yang, High actuated performance MWCNT/Ecoflex dielectric elastomer actuators based on layer-by-layer structure, Composites Part A: Applied Science and Manufacturing 125 (2019). doi:10.1016/j.compositesa.2019. 105527.

[23] J.-W. Lee, J. Chung, M.-Y. Cho, S. Timilsina, K. Sohn, J. S. Kim, K.-S. Sohn, Deep-Learning Technique To Convert a Crude Piezoresistive Carbon Nanotube-Ecoflex Composite Sheet into a Smart, Portable, Disposable, and Extremely Flexible Keypad, ACS Applied Materials \& Interfaces 10 (24) (2018) 20862-20868. doi:10.1021/acsami.8b04914.

URL https://pubs.acs.org/doi/10.1021/acsami.8b04914

[24] S. J. Kim, S. Mondal, B. K. Min, C.-G. Choi, Highly Sensitive and Flexible Strain-Pressure Sensors with Cracked Paddy-Shaped MoS 2 /Graphene Foam/Ecoflex Hybrid Nanostructures, ACS Applied Materials \& Interfaces 10 (42) (2018) 36377-36384. doi:10.1021/acsami . 8b11233. URL https://pubs.acs.org/doi/10.1021/acsami.8b11233

[25] R. U. R. Sagar, H. I. A. Qazi, M. H. Zeb, F. J. Stadler, B. Shabbir, X. Wang, M. Zhang, Tunable sign of magnetoresistance in graphene foam - Ecoflex ${ }^{\circledR}$ composite for wearable magnetoelectronic devices, Materials Letters 253 (2019) 166-170. doi:10.1016/j.matlet.2019.06.066.

[26] Y. Zhao, M. Ren, Y. Shang, J. Li, S. Wang, W. Zhai, G. Zheng, K. Dai, C. Liu, C. Shen, Ultrasensitive and durable strain sensor with sandwich structure and excellent anti-interference ability for wearable electronic skins, Composites Science and Technology 200 (2020). doi:10.1016/j. compscitech.2020.108448. 
[27] I. Spiridon, N. C. Anghel, R. N. Darie-Nita, A. Iwańczuk, R. G. Ursu, I. A. Spiridon, New composites based on starch/Ecoflex ${ }^{\circledR} /$ biomass wastes: Mechanical, thermal, morphological and antimicrobial properties, International Journal of Biological Macromolecules 156 (2020) 1435-1444. doi:10.1016/j.ijbiomac.2019.11.185.

[28] J. Yu, X. Hou, M. Cui, N. Zhang, S. Zhang, J. He, X. Chou, Skin-conformal BaTiO3/ecoflex-based piezoelectric nanogenerator for self-powered human motion monitoring, Materials Letters 269 (2020). doi:10.1016/j.matlet.2020.127686.

[29] L. Mullins, Effect of Stretching on the Properties of Rubber, Rubber Chemistry and Technology 21 (2) (1948) 281-300. doi:10.5254/1.3546914.

URL https://meridian.allenpress.com/rct/article/21/2/281/87705/ Effect-of-Stretching-on-the-Properties-of-Rubber

[30] D. E. Hanson, M. Hawley, R. Houlton, K. Chitanvis, P. Rae, E. B. Orler, D. A. Wrobleski, Stress softening experiments in silica-filled polydimethylsiloxane provide insight into a mechanism for the Mullins effect, Polymer 46 (24) (2005) 10989-10995. doi:10.1016/j.polymer.2005.09. 039.

[31] J. A. Harwood, A. R. Payne, Stress softening in natural rubber vulcanizates. Part III. Carbon blackfilled vulcanizates, Journal of Applied Polymer Science 10 (2) (1966) 315-324. doi:10.1002/ app.1966.070100212.

[32] Z. Liao, M. Hossain, X. Yao, R. Navaratne, G. Chagnon, A comprehensive thermo-viscoelastic experimental investigation of Ecoflex polymer, Polymer Testing 86 (March) (2020) 106478. doi: $10.1016 / j \cdot$ polymertesting.2020.106478. URL https://doi.org/10.1016/j.polymertesting.2020.106478

[33] V. Morovati, R. Dargazany, Micro-mechanical modeling of the stress softening in double-network hydrogels, ASME International Mechanical Engineering Congress and Exposition, Proceedings (IMECE) 9 (2018). doi : 10.1115/IMECE2018-88252.

[34] S. Wang, S. A. Chester, Modeling thermal recovery of the Mullins effect, Mechanics of Materials 126 (May) (2018) 88-98. doi:10.1016/j.mechmat.2018.08.002.

[35] G. Machado, G. Chagnon, D. Favier, Analysis of the isotropic models of the Mullins effect based on filled silicone rubber experimental results, Mechanics of Materials 42 (9) (2010) 841-851. doi: $10.1016 / j$.mechmat.2010.07.001.

URL https://linkinghub.elsevier.com/retrieve/pii/s0167663610000815

[36] J. Diani, B. Fayolle, P. Gilormini, A review on the Mullins effect, European Polymer Journal 45 (3) (2009) 601-612. doi:10.1016/j.eurpolymj.2008.11.017.

URL http://dx.doi.org/10.1016/j.eurpolymj.2008.11.017

[37] A. Andriyana, M. S. Loo, G. Chagnon, E. Verron, S. Y. Ch'Ng, Modeling the Mullins effect in elastomers swollen by palm biodiesel, International Journal of Engineering Science 95 (2015) 1-22. doi:10.1016/j.ijengsci.2015.06.005. 
[38] Z. Liao, M. Hossain, X. Yao, Ecoflex polymer of different Shore hardnesses: Experimental investigations and constitutive modelling, Mechanics of Materials 144 (January) (2020). doi:10.1016/j. mechmat.2020.103366.

[39] G. MacHado, G. Chagnon, D. Favier, Induced anisotropy by the Mullins effect in filled silicone rubber, Mechanics of Materials 50 (2012) 70-80. doi:10.1016/j.mechmat.2012.03.006.

URL http://dx.doi.org/10.1016/j.mechmat.2012.03.006

[40] Z. Rigbi, Reinforcement of Rubber by Carbon Black, Rubber Chemistry and Technology 55 (4) (1982) 1180-1220. doi:10.5254/1.3535922.

URL https://meridian.allenpress.com/rct/article/55/4/1180/91635/ Reinforcement-of-Rubber-by-Carbon-Black

[41] L. Yan, D. A. Dillard, R. L. West, L. D. Lower, G. V. Gordon, Mullins effect recovery of a nanoparticlefilled polymer, Journal of Polymer Science, Part B: Polymer Physics 48 (21) (2010) 2207-2214. do i : $10.1002 /$ polb.22102.

[42] S. Y. Zheng, H. Ding, J. Qian, J. Yin, Z. L. Wu, Y. Song, Q. Zheng, Metal-Coordination Complexes Mediated Physical Hydrogels with High Toughness, Stick-Slip Tearing Behavior, and Good Processability, Macromolecules 49 (24) (2016) 9637-9646. doi:10 .1021/acs . macromol . 6b 02150.

[43] F. Laraba-Abbesa, P. Ienny, R. Piques, A new 'tailor-made' methodology for the mechanical behaviour analysis of rubber-like materials: II. Application to the hyperelastic behaviour characterization of a carbon-black filled natural rubber vulcanizate, Polymer 44 (3) (2002) 821-840. doi:10.1016/ S0032-3861(02)00719-X.

[44] A. F. Amin, A. Lion, P. Höfer, Effect of temperature history on the mechanical behaviour of a fillerreinforced NR/BR blend: Literature review and critical experiments, ZAMM Zeitschrift fur Angewandte Mathematik und Mechanik 90 (5) (2010) 347-369. doi:10.1002/ zamm. 200900365.

[45] J. Plagge, M. Klüppel, Mullins effect revisited: Relaxation, recovery and high-strain damage, Materials Today Communications 20 (July) (2019). doi:10.1016/j.mtcomm.2019.100588.

[46] R. Diaz, J. Diani, P. Gilormini, Physical interpretation of the Mullins softening in a carbon-black filled SBR, Polymer 55 (19) (2014) 4942-4947. doi : $10.1016 / j$.polymer. 2014.08 .020$.

URL http://dx.doi.org/10.1016/j.polymer.2014.08.020

[47] Z. Li, H. Xu, X. Xia, Y. Song, Q. Zheng, Energy dissipation accompanying Mullins effect of nitrile butadiene rubber/carbon black nanocomposites, Polymer 171 (January) (2019) 106-114. doi:10 . $1016 / j \cdot$ polymer.2019.03.043.

URL https://doi.org/10.1016/j.polymer.2019.03.043https://linkinghub. elsevier.com/retrieve/pii/s0032386119302678

[48] Z. Li, F. Wen, M. Hussain, Y. Song, Q. Zheng, Scaling laws of Mullins effect in nitrile butadiene rubber nanocomposites, Polymer 193 (2020). doi:10.1016/j.polymer.2020.122350.

[49] A. D. Drozdov, A. Dorfmann, Finite viscoelasticity of filled rubbers: The effects of pre-loading and thermal recovery, Continuum Mechanics and Thermodynamics 14 (4) (2002) 337-361. arXiv: 0110269, doi:10.1007/s001610100073. 
[50] H. Chu, J. Lin, D. Lei, J. Qian, R. Xiao, A network evolution model for recovery of the mullins effect in filled rubbers, International Journal of Applied Mechanics 12 (9) (2020) 1-16. doi:10.1142/ S1758825120501082.

[51] J. L. Sparks, N. A. Vavalle, K. E. Kasting, B. Long, M. L. Tanaka, P. A. Sanger, K. Schnell, T. A. Conner-Kerr, Use of silicone materials to simulate tissue biomechanics as related to deep tissue injury, Advances in Skin and Wound Care 28 (2) (2015) 59-68. doi : 10.1097/01. A.SW. 0000460127. $47415.6 e$.

[52] S. Park, K. Mondal, R. M. Treadway, V. Kumar, S. Ma, J. D. Holbery, M. D. Dickey, Silicones for Stretchable and Durable Soft Devices: Beyond Sylgard-184, ACS Applied Materials \& Interfaces 10 (13) (2018) 11261-11268. doi:10.1021/acsami. 7b18394.

URL https://pubs .acs.org/doi/10.1021/acsami.7b18394

[53] J. Vaicekauskaite, P. Mazurek, S. Vudayagiri, A. L. Skov, Mapping the mechanical and electrical properties of commercial silicone elastomer formulations for stretchable transducers, Journal of Materials Chemistry C 8 (4) (2020) 1273-1279. doi : $10.1039 /$ C9TC05072H.

URL http://xlink.rsc.org/?DOI=C9TC05072H

[54] C. S. Woo, W. D. Kim, Design of mechanical testing specimens for rubber material using finite element analysis, Multidiscipline Modeling in Materials and Structures 3 (3) (2007) 325-336. doi:10.1163/157361107781389535.

[55] I. Axel Products, Testing Brief: Compression or Biaxial Extension? 1-3.

URL http: / / www . axelproducts.com/downloads/CompressionorBiax.pdf

[56] J. Day, K. Miller, Equibiaxial Stretching of Elastomeric Sheets: An Analytical Verification of Experimental Technique, ABAQUS 2000 User's Conference Proceedings 21 (2000) 205-220.

URL http: / /www.axelproducts.com/downloads/BiaxialExtension.pdf

[57] R. Xiao, T. T. Mai, K. Urayama, J. P. Gong, S. Qu, Micromechanical modeling of the multi-axial deformation behavior in double network hydrogels, International Journal of Plasticity (November) (2020) 102901. doi:10.1016/j.ijplas.2020.102901.

URL https://doi.org/10.1016/j.ijplas.2020.102901

[58] C. Zhang, X. Gou, R. Xiao, Hysteresis in glass microsphere filled elastomers under cyclic loading, Polymer Testing 95 (xxxx) (2021) 107081. doi:10.1016/j.polymertesting.2021. 107081.

URL https://doi.org/10.1016/j.polymertesting.2021.107081

[59] Z. Liao, M. Hossain, X. Yao, M. Mehnert, P. Steinmann, On thermo-viscoelastic experimental characterization and numerical modelling of VHB polymer, International Journal of Non-Linear Mechanics 118 (2020) 103263. doi:10.1016/j.ijnonlinmec.2019.103263.

URL https://linkinghub.elsevier.com/retrieve/pii/s0020746219303415

[60] Y. Chen, G. Kang, J. Yuan, T. Li, Experimental study on pure-shear-like cyclic deformation of VHB 4910 dielectric elastomer, Journal of Polymer Research 26 (8) (2019) 186. doi:10.1007/ s10965-019-1858-6.

URL http://link.springer.com/10.1007/s10965-019-1858-6 
[61] A. Bojtos, A. Huba, L. Zentner, U. Risto, Measuring of equibiaxial tension of rubber-like materials by optical method, Materials Science Forum 659 (2010) 289-294. doi:10.4028/www .

[62] T.-t. Mai, K. Urayama, Biaxial Loading Effects on Strain Energy Release Rate and Crack-Tip Strain Field in Elastic Hydrogels, Macromolecules 54 (10) (2021) 4792-4801. doi:10.1021/acs. macromol.1c00445.

URL https://pubs.acs.org/doi/10.1021/acs.macromol.1c00445

[63] T. Aoyama, N. Yamada, K. Urayama, Nonlinear Elasticity of Ultrasoft Near-Critical Gels with Extremely Sparse Network Structures Revealed by Biaxial Stretching, Macromolecules 54 (5) (2021) 2353-2365. doi:10.1021/acs.macromol.0c02737.

URL https://pubs.acs.org/doi/10.1021/acs.macromol.0c02737

[64] S. Krpovic, K. Dam-Johansen, A. L. Skov, Importance of Mullins effect in commercial silicone elastomer formulations for soft robotics, Journal of Applied Polymer Science (December) (2020) 1-15. doi:10.1002/app.50380.

[65] A. M. Stricher, R. G. Rinaldi, C. Barrès, F. Ganachaud, L. Chazeau, How I met your elastomers: from network topology to mechanical behaviours of conventional silicone materials, RSC Advances 5 (66) (2015) 53713-53725. doi:10.1039/c5ra06965c.

[66] P. Mazurek, S. Vudayagiri, A. L. Skov, How to tailor flexible silicone elastomers with mechanical integrity: A tutorial review, Chemical Society Reviews 48 (6) (2019) 1448-1464. doi:10.1039/ c8cs00963e.

[67] A. L. Skov, L. Yu, Optimization Techniques for Improving the Performance of Silicone-Based Dielectric Elastomers, Advanced Engineering Materials 20 (5) (2018) 1700762. doi:10.1002/adem. 201700762.

URL http://doi.wiley.com/10.1002/adem.201700762

[68] I. A. Morozov, L. A. Komar, B. Lauke, Structural-mechanical model of filled rubber: Influence of filler arrangement, International Journal of Mechanical Sciences 107 (2016) 160-169. doi : $10.1016 / j$. ijmecsci.2016.01.013.

[69] L. Mullins, N. R. Tobin, Theoretical Model for the Elastic Behavior of Filler-Reinforced Vulcanized Rubbers, Rubber Chemistry and Technology 30 (2) (1957) 555-571. doi:10.5254/1.3542705. URL https://meridian.allenpress.com/rct/article/30/2/555/90092/ Theoretical-Model-for-the-Elastic-Behavior-of

[70] J. S. Bergström, M. C. Boyce, Mechanical behavior of particle filled elastomers, Rubber Chemistry and Technology 72 (4) (1999) 633-656. doi:10.5254/1.3538823.

URL https://meridian.allenpress.com/rct/article/72/4/633/92838/ Mechanical-Behavior-of-Particle-Filled-Elastomers

[71] S. Trabelsi, P. A. Albouy, J. Rault, Effective Local Deformation in Stretched Filled Rubber, Macromolecules 36 (24) (2003) 9093-9099. doi:10 .1021/ma 0303566. 
[74] Y. Song, Q. Zheng, A Guide for Hydrodynamic Reinforcement Effect in Nanoparticle-filled Polymers, Critical Reviews in Solid State and Materials Sciences 41 (4) (2016) 318-346. doi:10.1080/ 10408436.2015 .1135415$. 